\title{
Polyoxometalate-Driven Ease Conversion of Valuable Furfural to trans-N,N-4,5-Diaminocyclopenten-2-ones
}

Marina A. Tzani, Stella Fountoulaki, and Ioannis N. Lykakis*

Department of Chemistry, Aristotle University of Thessaloniki, University Campus 54124,

Thessaloniki, Greece; E-mail: lykakis@chem.auth.gr

\begin{tabular}{|c|c|}
\hline Page & Table of contents \\
\hline S2-S11 & Evaluation of the catalytic reactions \\
\hline S12-S14 & Copies of ${ }^{1} \mathrm{H}$ and ${ }^{13} \mathrm{C}\{\mathrm{H}\}$ NMR spectra of synthetic secondary amines \\
\hline S16-S36 & Copies of ${ }^{1} \mathrm{H}$ and ${ }^{13} \mathrm{C}\{\mathrm{H}\}$ NMR spectra of trans-DACPs from secondary amines \\
\hline S37-S46 & Copies of ${ }^{1} \mathrm{H}$ and ${ }^{13} \mathrm{C}\{\mathrm{H}\}$ NMR spectra of trans-DACPs from substituted anilines \\
\hline
\end{tabular}


Table S1. Reaction's profile in the absence of a catalyst.

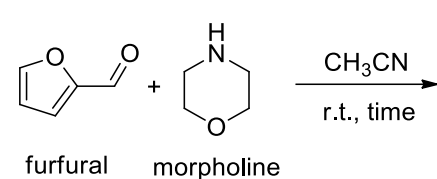

(1)

(2)

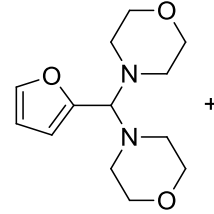

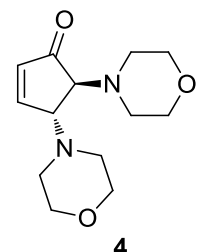

\begin{tabular}{cccccc}
\hline Entry & Time $^{\mathrm{a}}$ & ${\text { Conversion }(\boldsymbol{\%})^{\mathrm{b}}}$ & $\mathbf{1}(\boldsymbol{\%})^{\mathrm{c}}$ & $\mathbf{3}(\boldsymbol{\%})^{\mathrm{c}}$ & $\mathbf{4}(\boldsymbol{\%})^{\mathrm{c}}$ \\
\hline $\mathbf{1}$ & $1 \mathrm{~h}$ & 82 & 18 & 58 & 24 \\
$\mathbf{2}$ & $3 \mathrm{~h}$ & 90 & 10 & 68 & 22 \\
$\mathbf{3}$ & $6 \mathrm{~h}$ & 88 & 12 & 54 & 34 \\
$\mathbf{4}$ & $18 \mathrm{~h}$ & 88 & 12 & 44 & 44
\end{tabular}

${ }^{\mathrm{a}}$ Reaction Conditions: $1(0.5 \mathrm{mmol}), 2(1 \mathrm{mmol})$ and acetonitrile $(0.5$ $\mathrm{ml})$ at room temperature. ${ }^{\mathrm{b}}$ Based on the consumption of $\mathbf{1}$ determined from the crude ${ }^{1} \mathrm{H}$ NMR mixture of the reaction. ${ }^{\mathrm{c}}$ Relative yields of $\mathbf{3}$ and 4 were determined by ${ }^{1} \mathrm{H}$ NMR from the crude reaction mixture. 


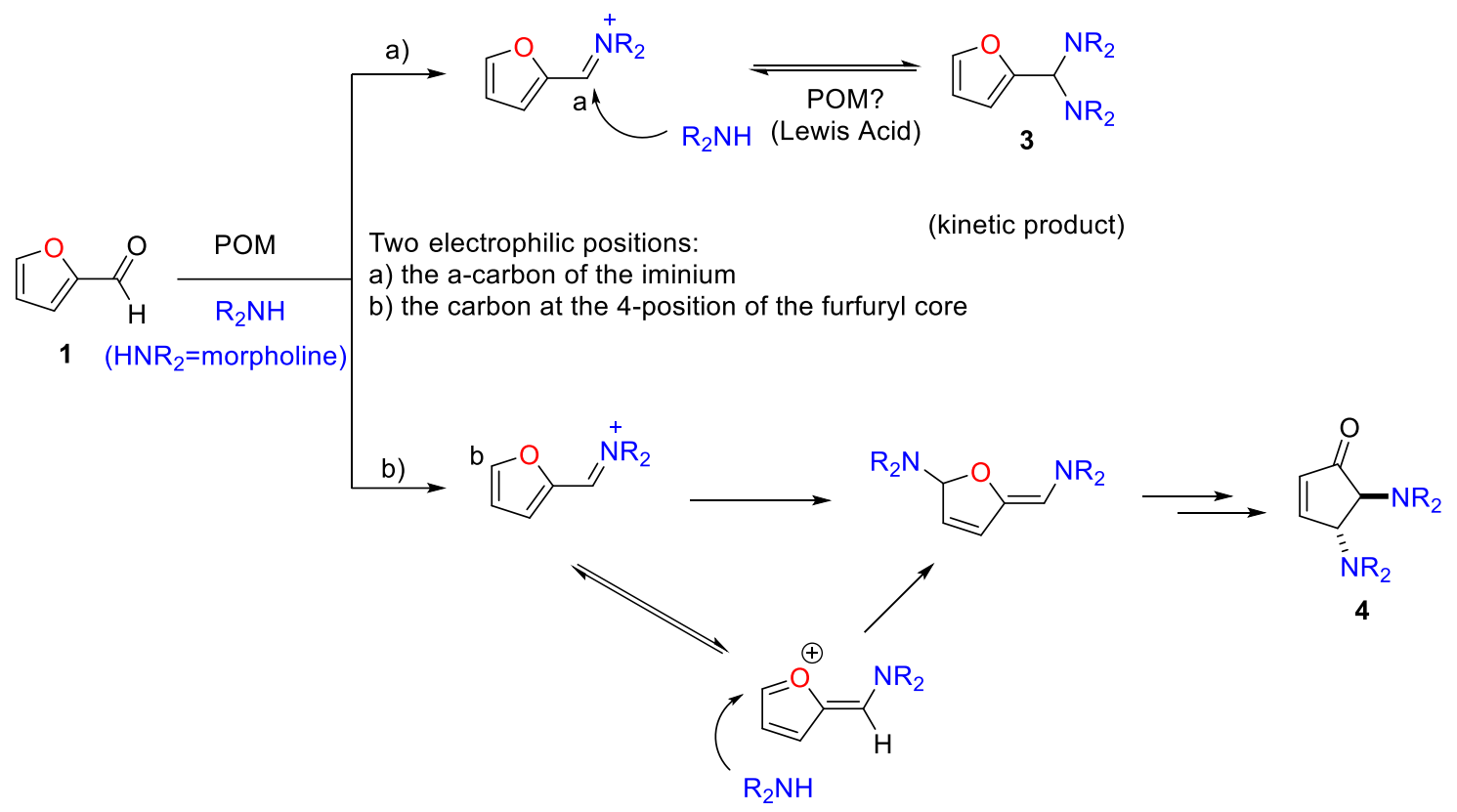

Scheme S1. Plausible reaction paths for the transformation of 3 to 4 . 



Figure S1. ${ }^{1} \mathrm{H},{ }^{13} \mathrm{C}$ and COSY NMR spectra $\left(500 \mathrm{MHz}, \mathrm{CDCl}_{3}\right)$ for compound 3. 
Table S2. Solvent evaluation in the absence of a catalyst.
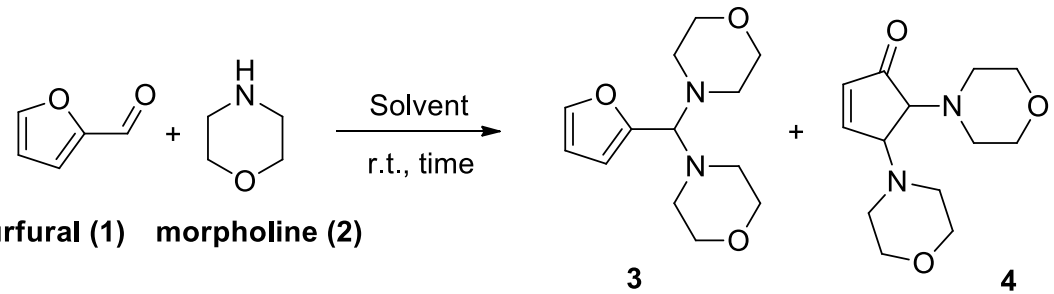

furfural (1) morpholine (2)

3

4

\begin{tabular}{|c|c|c|c|c|c|}
\hline Entry & Solvent $^{\mathrm{a}}$ & Time (h) & $1(\%)^{b}$ & $3(\%)^{c}$ & $4(\%)^{c}$ \\
\hline 1 & DCE & 6 & 23 & 37 & 40 \\
\hline 2 & DCE & 18 & 11 & 48 & 41 \\
\hline 3 & EtOAc & 6 & 28 & 34 & 38 \\
\hline 4 & EtOAc & 18 & 11 & 73 & 16 \\
\hline 5 & DMSO & 6 & 20 & 65 & 15 \\
\hline 6 & DMSO & 18 & 42 & 56 & 23 \\
\hline 7 & DMF & 6 & 21 & 37 & 42 \\
\hline 8 & DMF & 18 & 25 & 20 & 55 \\
\hline 9 & $\mathrm{DMC}$ & 6 & 18 & 59 & 23 \\
\hline 10 & DMC & 18 & 8 & 66 & 26 \\
\hline 11 & THF & 6 & 27 & 40 & 33 \\
\hline 12 & THF & 18 & 13 & 51 & 36 \\
\hline \multicolumn{6}{|c|}{$\begin{array}{l}\text { a Reaction's Conditions: furfural }(0.5 \mathrm{mmol}) \text {, morpholine }(1 \mathrm{mmol}) \text { and solvent }(0.5 \mathrm{ml}) \text { at } \\
\text { room temperature. }{ }^{b} \text { Based on the consumption of } \mathbf{1} \text { determined from the crude }{ }^{1} \mathrm{H} \text { NMR } \\
\text { mixture of the reaction. }{ }^{\mathrm{c}} \text { Relative yields of } \mathbf{3} \text { and } \mathbf{4} \text { were determined by }{ }^{1} \mathrm{H} \text { NMR from the } \\
\text { crude reaction mixture. }\end{array}$} \\
\hline
\end{tabular}





$11,79 \%$

12, $80 \%$

$13,69 \%$

14, $73 \%$

$15,81 \%$<smiles>CCCCNCc1ccc(Cl)cc1</smiles>

16, $96 \%$

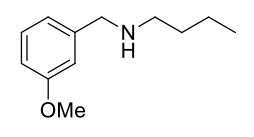

$17,84 \%$

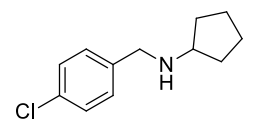

$18,90 \%$<smiles>Cc1ccc(CNC2CCCC2)cc1</smiles>

19, $87 \%$

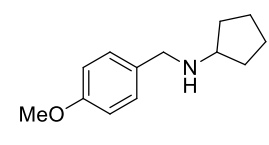

$20,93 \%$

Scheme S2. Synthesis of secondary amines. 

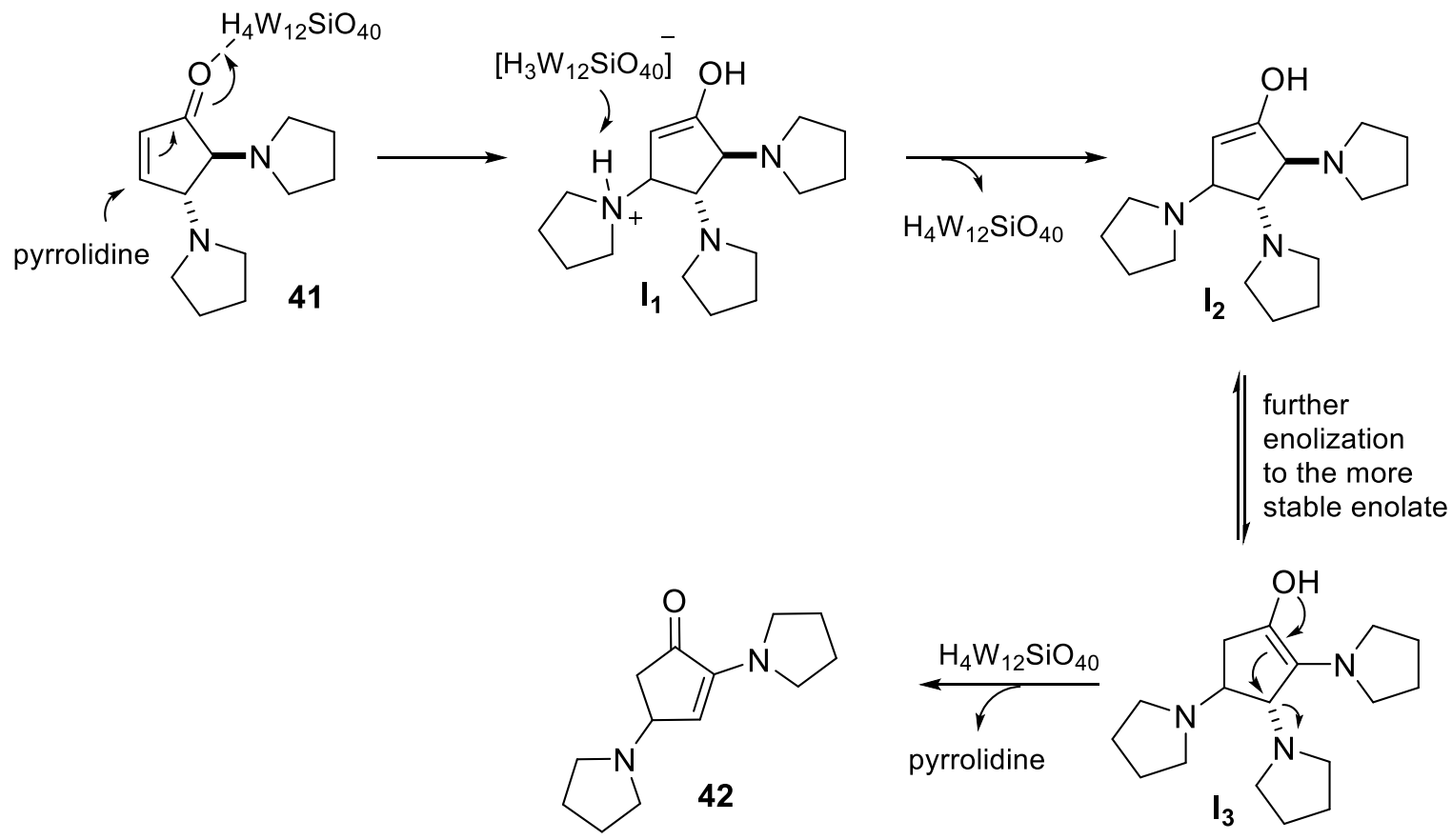

Scheme S3. Plausible pathway of the transformation of $\mathbf{4 1}$ to $\mathbf{4 2}$ in the presence of silicotungstic acid. 
Table S3. POM-catalysts screening to the selective synthesis of 4,5-bis((4-

chlorophenyl)amino)cyclopent-2-enone 49.

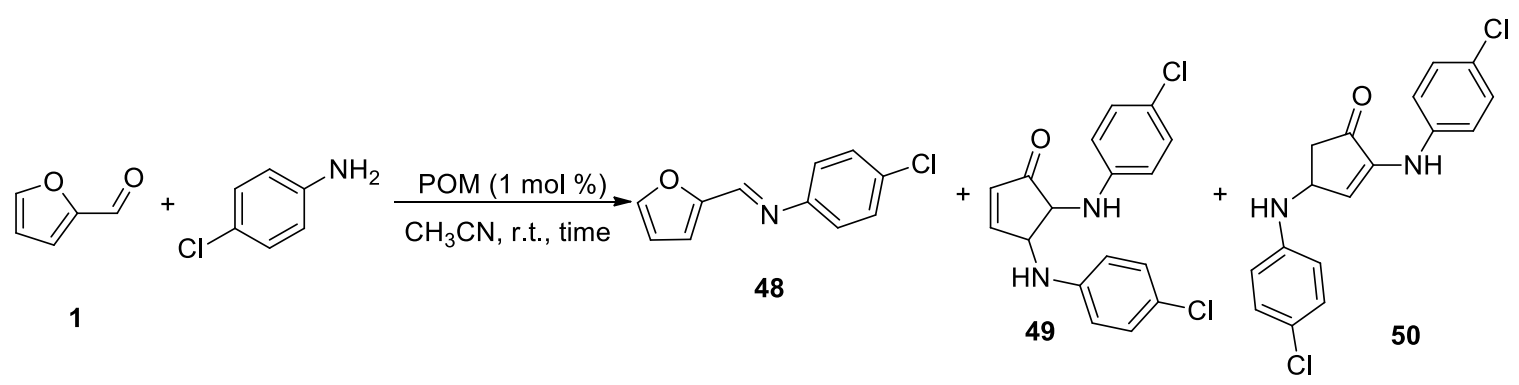

\begin{tabular}{cccccc}
\hline Entry & POM $(\mathbf{1} \text { mol\% })^{\mathbf{a}}$ & Time & $\mathbf{4 8}(\boldsymbol{\%})^{\mathbf{b}}$ & $\mathbf{4 9}(\boldsymbol{\%})^{\mathbf{b}}$ & $\mathbf{5 0}(\boldsymbol{\%})^{\mathbf{b}}$ \\
\hline 1 & $\mathrm{H}_{4} \mathrm{SiW}_{12} \mathrm{O}_{40}$ & $4 \mathrm{~h}$ & 10 & 90 & - \\
2 & $\mathrm{H}_{3} \mathrm{PMo}_{12} \mathrm{O}_{40}$ & $4 \mathrm{~h}$ & 27 & 73 & - \\
3 & $\mathrm{H}_{3} \mathrm{PW}_{12} \mathrm{O}_{40}$ & $4 \mathrm{~h}$ & 21 & 79 & - \\
4 & $\mathrm{Na}_{3} \mathrm{PW}_{12} \mathrm{O}_{40}$ & $4 \mathrm{~h}$ & 67 & 33 & - \\
5 & $\mathrm{No} \mathrm{catalyst}_{4}$ & $4 \mathrm{~h}$ & 59 & 34 & 7 \\
6 & $\mathrm{H}_{4} \mathrm{SiW}_{12} \mathrm{O}_{40}$ & $18 \mathrm{~h}$ & - & 90 & 10 \\
7 & $\mathrm{H}_{3} \mathrm{PMo}_{12} \mathrm{O}_{40}$ & $18 \mathrm{~h}$ & 8 & 31 & 61 \\
8 & $\mathrm{H}_{3} \mathrm{PW}_{12} \mathrm{O}_{40}$ & $18 \mathrm{~h}$ & 6 & 42 & 52 \\
9 & $\mathrm{Na}_{3} \mathrm{PW}_{12} \mathrm{O}_{40}$ & $18 \mathrm{~h}$ & 15 & 54 & 31 \\
10 & $\mathrm{No} \mathrm{catalyst}_{0}$ & $18 \mathrm{~h}$ & 55 & 35 & 10 \\
\hline
\end{tabular}

${ }^{a}$ Reaction conditions: POM (1 mol \%), furfural $(0.5 \mathrm{mmol})$, 4-chloroaniline $(1 \mathrm{mmol})$ in acetonitrile $(0.5 \mathrm{ml})$ at room temperature. ${ }^{\mathrm{b}}$ Yields of $\mathbf{1}, \mathbf{4 8}, 49$ and 50 were determined by ${ }^{1} \mathrm{H}$ NMR from the crude reaction mixture. 


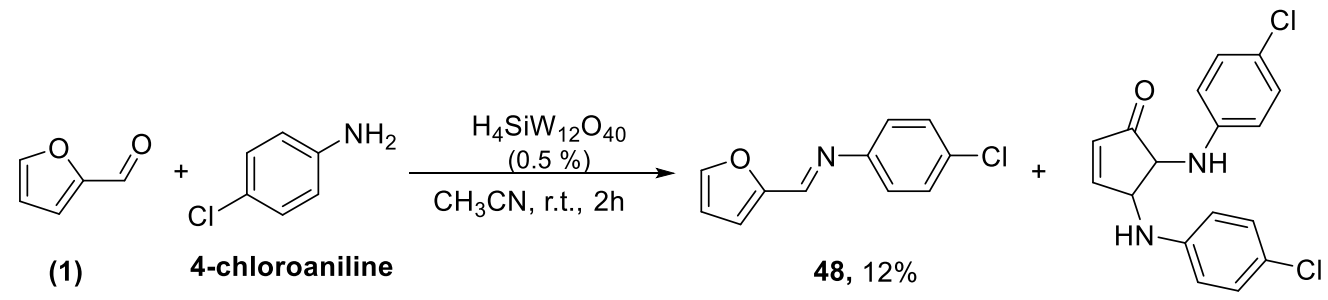

$49,88 \%$



Scheme S4. The catalytic reaction between furfural and 4-chloroaniline.



(1)<smiles>Clc1ccccc1/N=C/c1ccco1</smiles>

$60,15 \%$<smiles>O=C1C=CC(Nc2ccccc2Cl)C1Nc1ccccc1Cl</smiles>

51, $85 \%$ chromatography

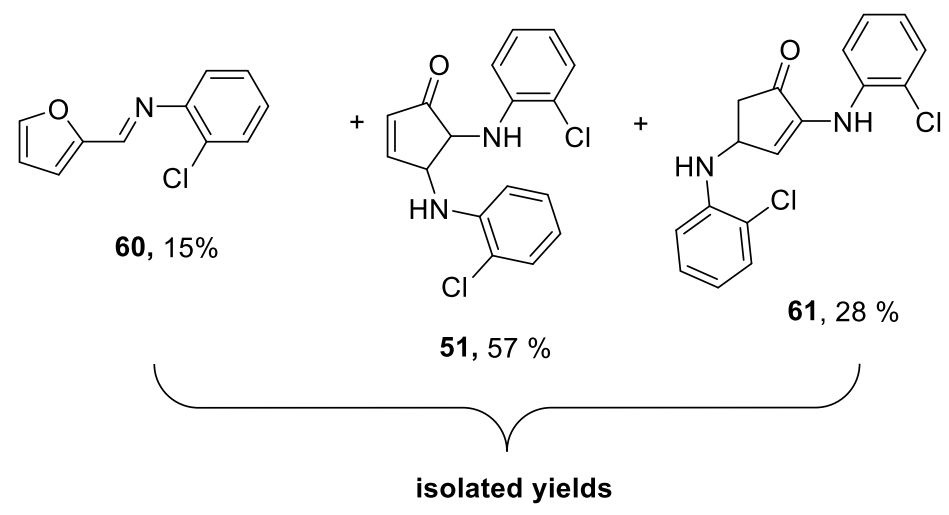

Scheme S5. The catalytic reaction between furfural and 2-chloroaniline. 


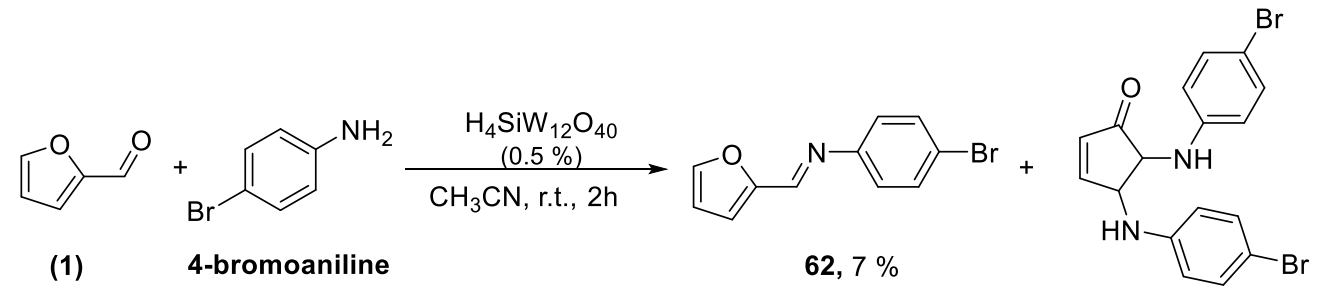

52, $93 \%$

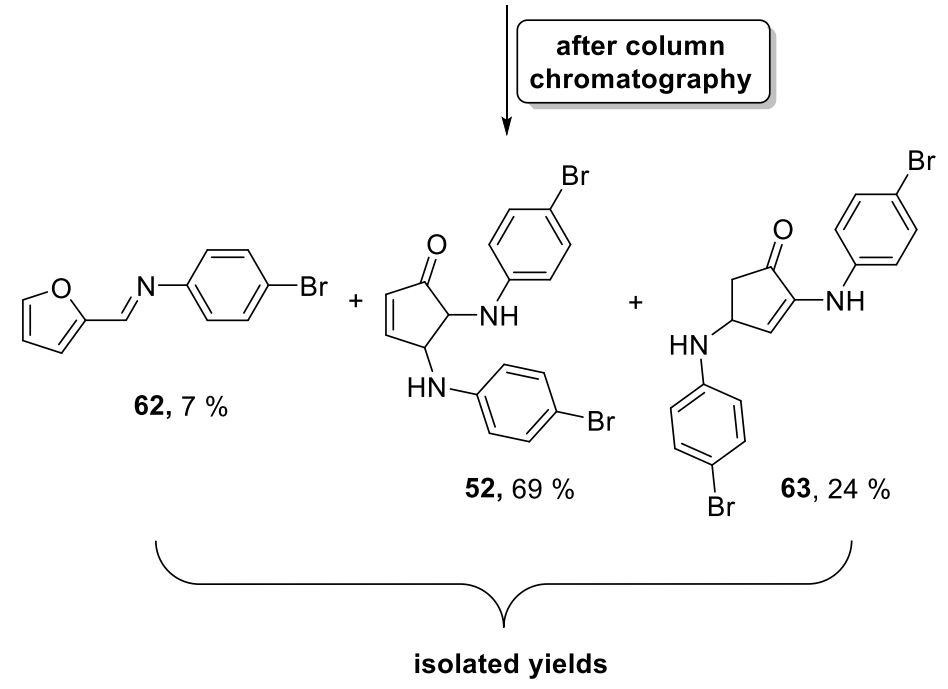

Scheme S6. The catalytic reaction between furfural and 4-bromoaniline. 
Table S4. Reports on the transformation of furfural into trans-DACPs with secondary and primary amines.



\begin{tabular}{cccccccc}
\hline Reports & Promoter & $\begin{array}{c}\text { Loading } \\
(\%)\end{array}$ & Solvent & Time & Temp. & $\begin{array}{c}\text { Secondary } \\
\text { Amines }\end{array}$ & $\begin{array}{c}\text { Primary } \\
\text { Amines }\end{array}$ \\
Batey, 2007 & Dy $(\mathrm{OTf})_{3}$ & 10 & $\mathrm{CH}_{3} \mathrm{CN}$ & $16 \mathrm{~h}$ & r.t & $\sqrt{ }$ & Aniline \\
[Sc $\left.(\mathrm{OTf})_{3}\right]$
\end{tabular}


Copies of ${ }^{1} \mathrm{H}$ and ${ }^{13} \mathrm{C}\{\mathrm{H}\}$ NMR spectra of synthetic secondary amines

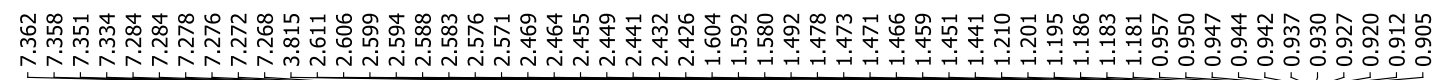<smiles>CCC(C)CNCc1ccccc1</smiles>

${ }^{1} \mathrm{H}$ NMR $\left(500 \mathrm{MHz}, \mathrm{CDCl}_{3}\right)$

9
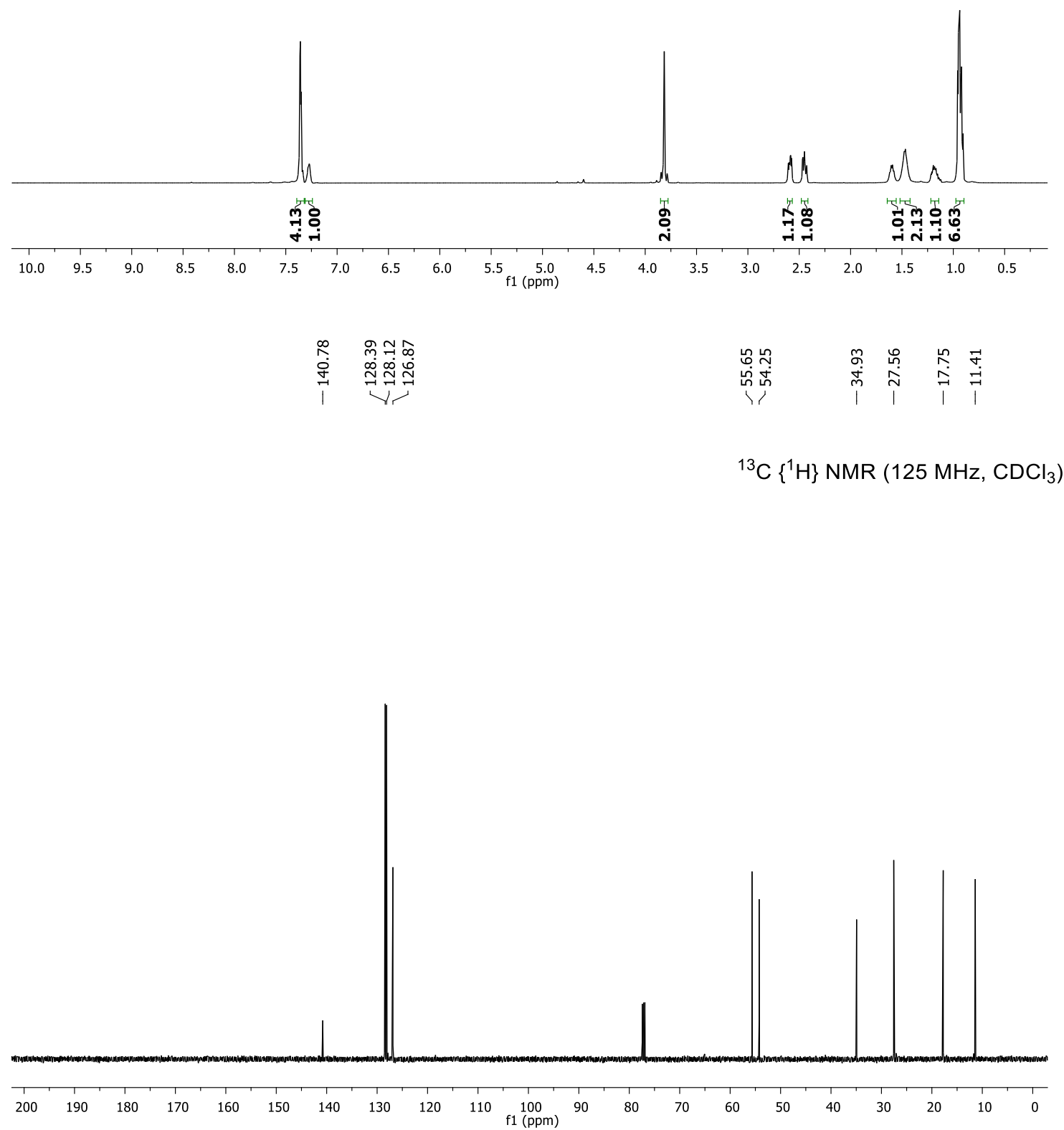

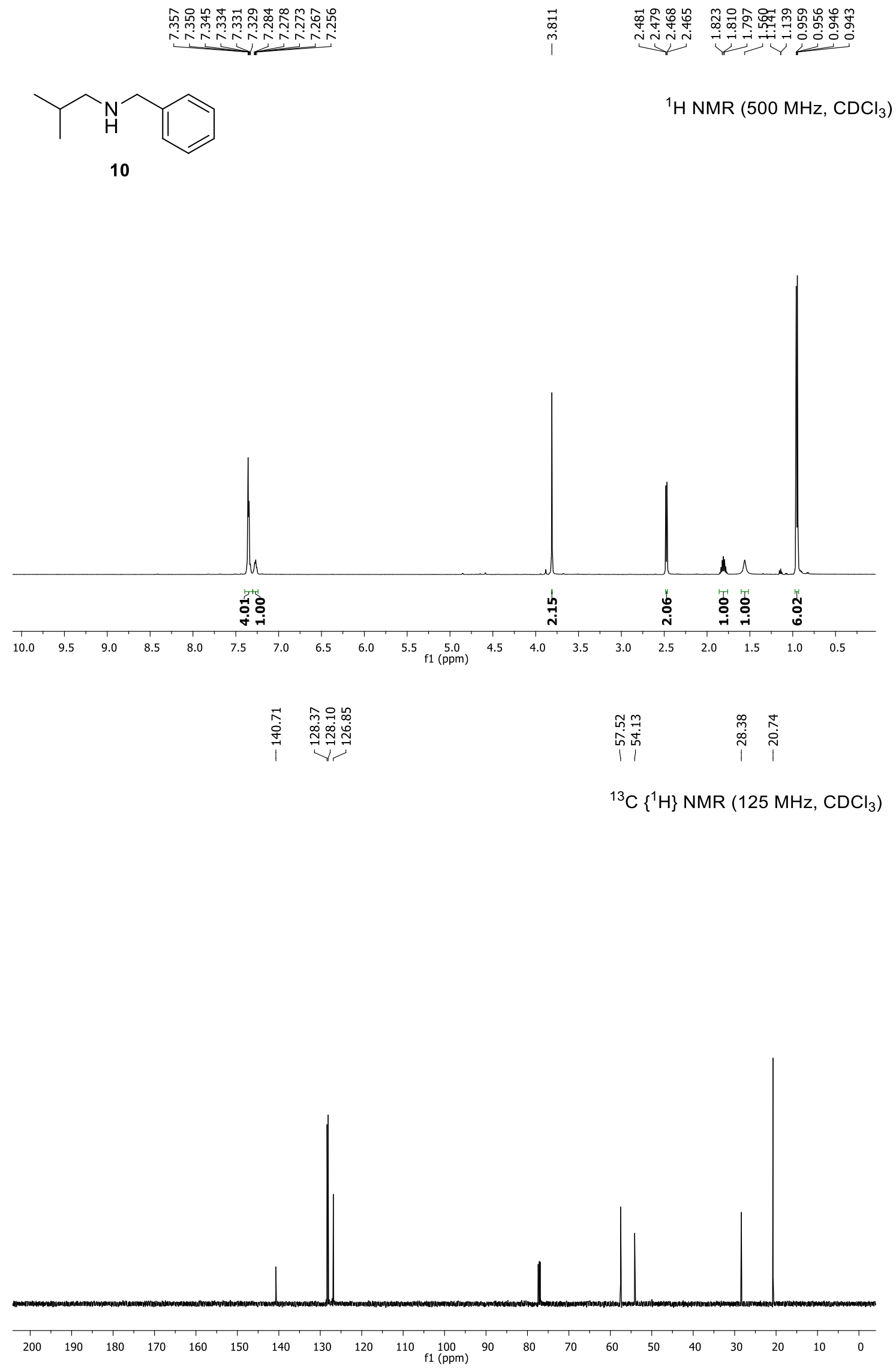

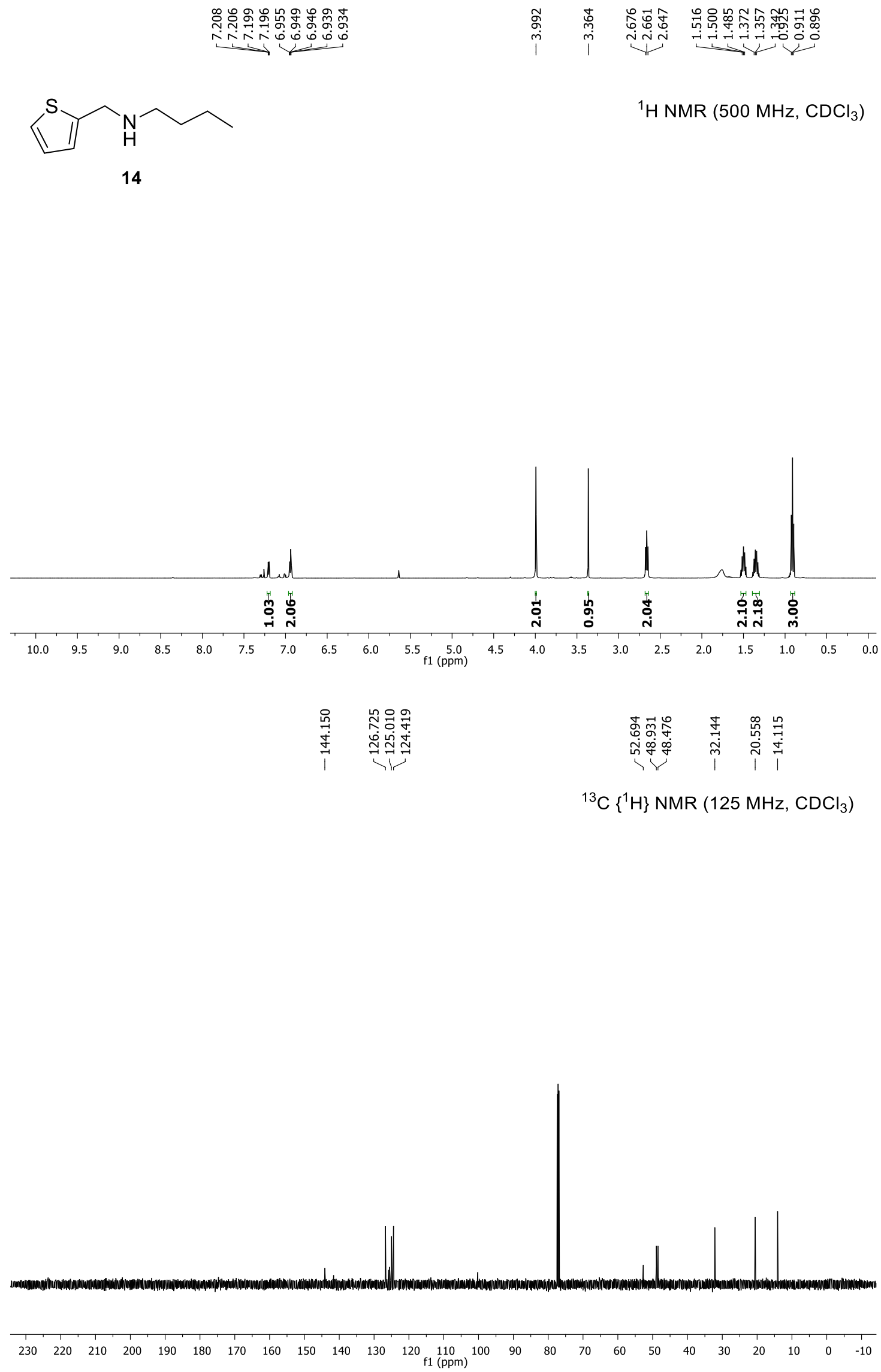

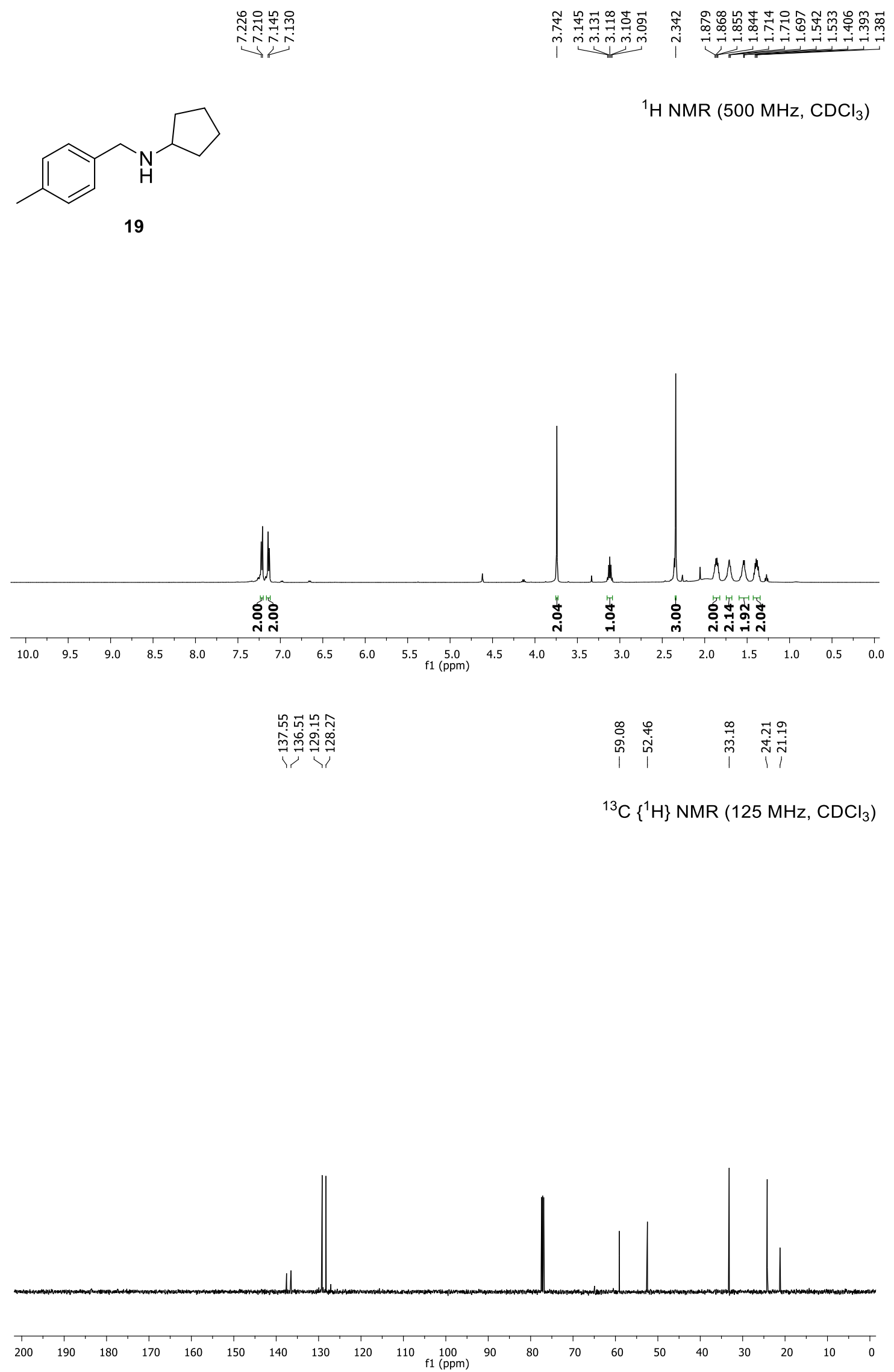
Copies of ${ }^{1} \mathrm{H}$ and ${ }^{13} \mathrm{C}\{\mathrm{H}\}$ NMR spectra of trans-DACPs from secondary amines

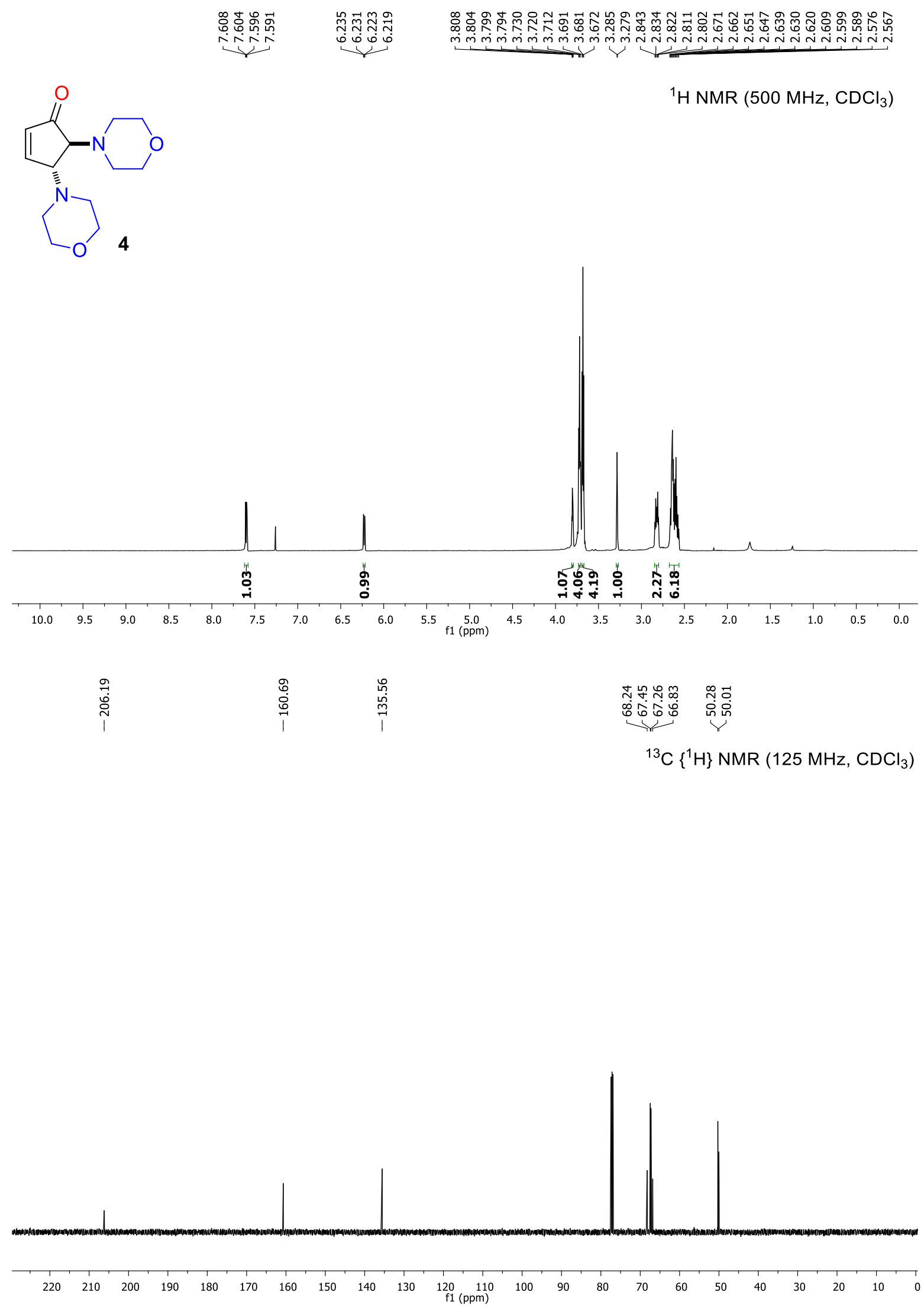


<smiles>O=C1C=C[C@@H](N2CCCCC2)[C@H]1N1CCCCC1</smiles>

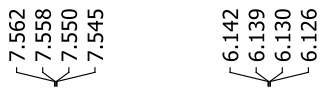



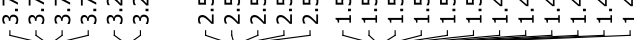

${ }^{1} \mathrm{H}$ NMR $\left(500 \mathrm{MHz}, \mathrm{CDCl}_{3}\right)$



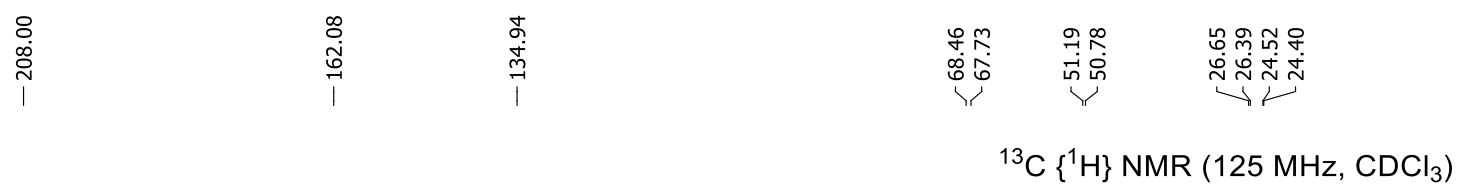

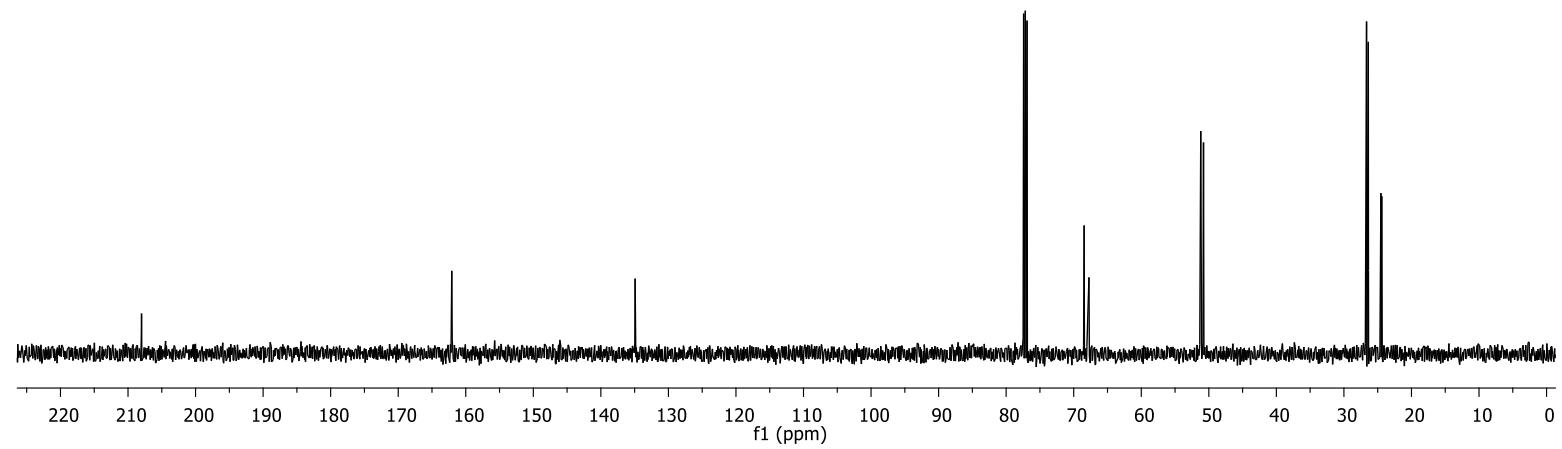




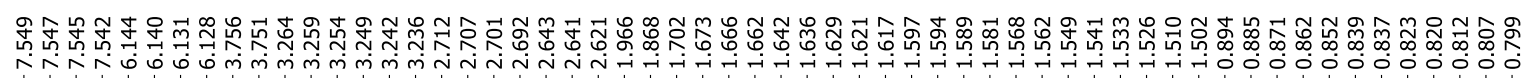<smiles>CC1CCCN(C2C(=O)C=C[C@@H]2N2CCCC(C)C2)C1</smiles>

${ }^{1} \mathrm{H} \mathrm{NMR}\left(500 \mathrm{MHz}, \mathrm{CDCl}_{3}\right)$



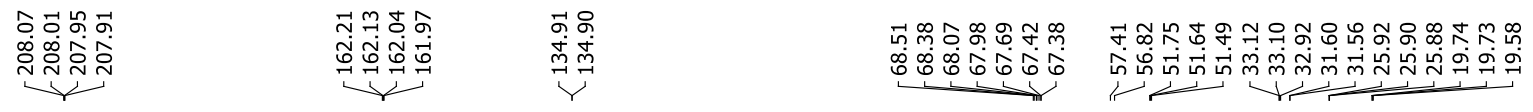

${ }^{13} \mathrm{C}\left\{{ }^{1} \mathrm{H}\right\}$ NMR $\left(125 \mathrm{MHz}, \mathrm{CDCl}_{3}\right)$






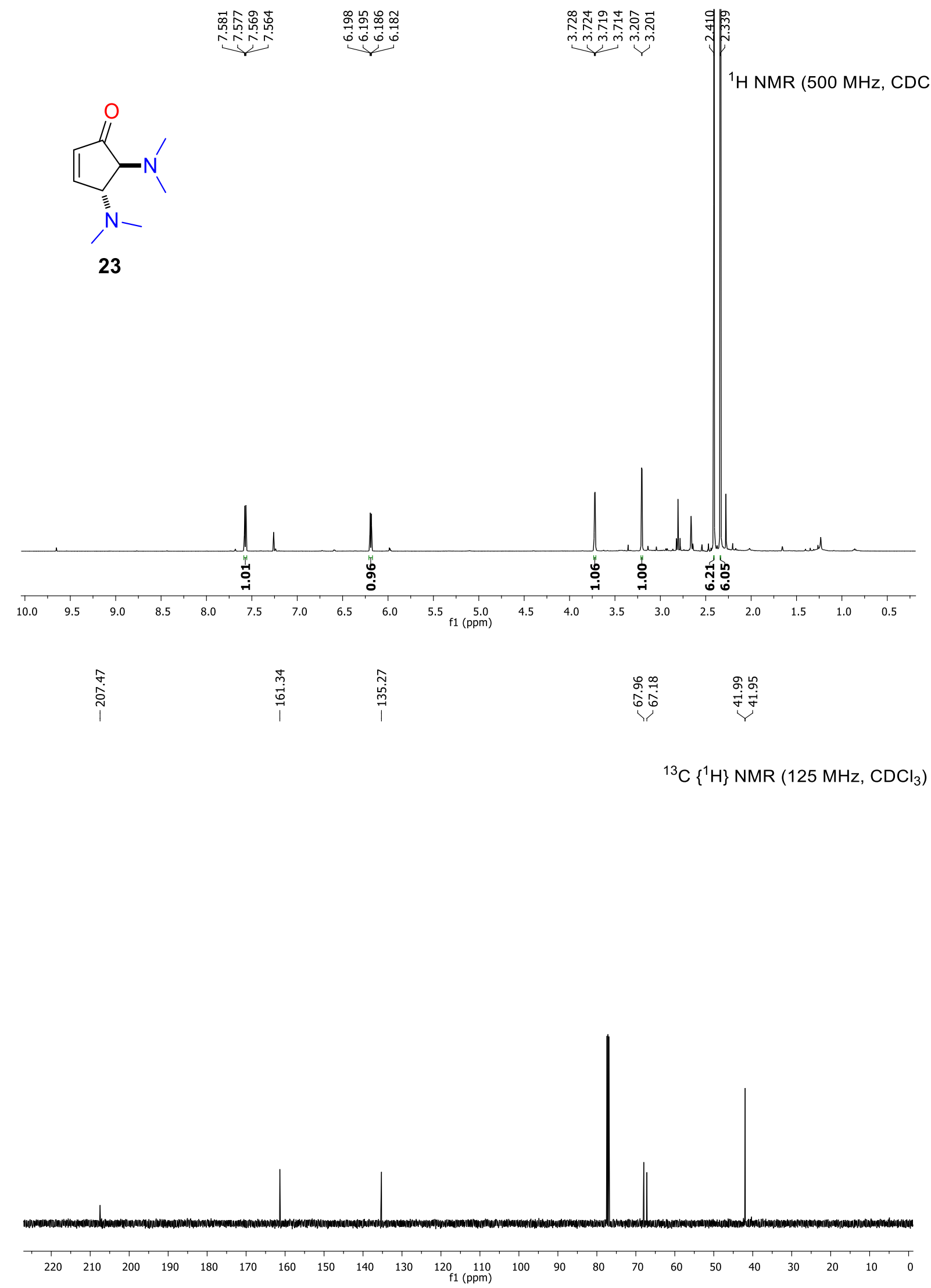




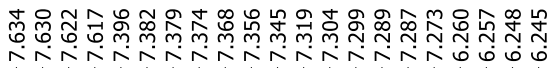<smiles>O=C1C=C[C@@H](N(Cc2ccccc2)Cc2ccccc2)N(Cc2ccccc2)C1</smiles>

24



ナ $\dot{m} \dot{m} \dot{m} \dot{m} \dot{m} \dot{m}$

${ }^{1} \mathrm{H}$ NMR $\left(500 \mathrm{MHz}, \mathrm{CDCl}_{3}\right)$

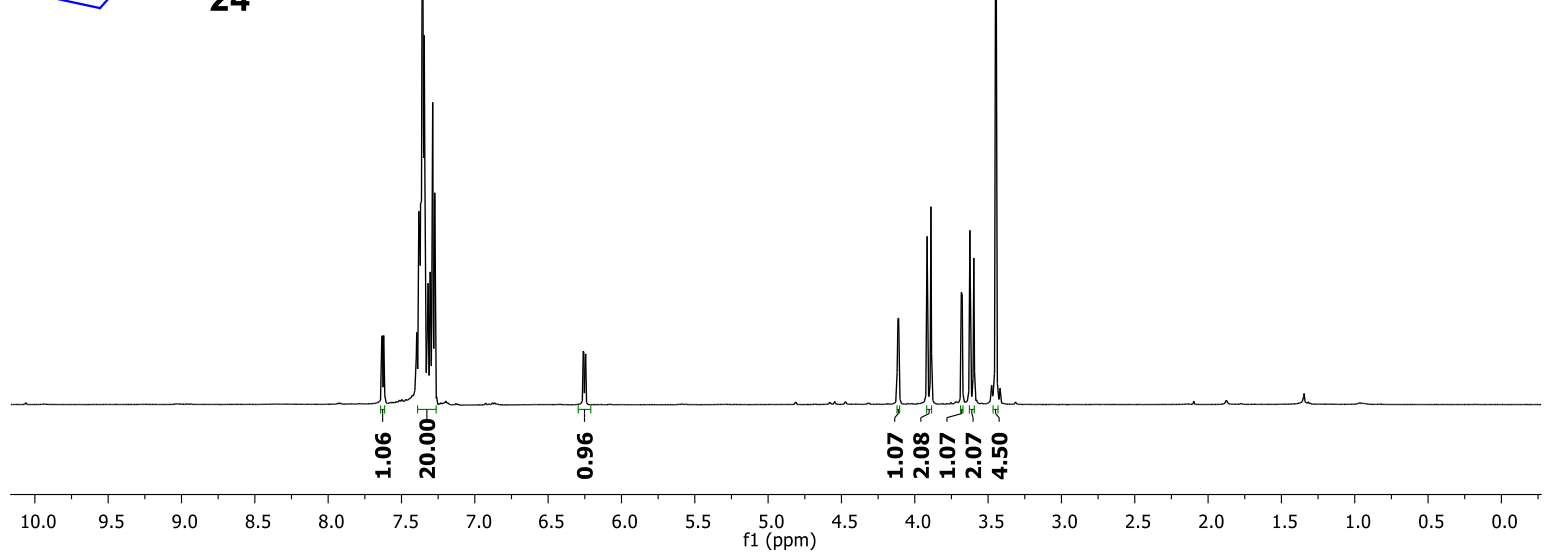



${ }^{13} \mathrm{C}\left\{{ }^{1} \mathrm{H}\right\}\left(125 \mathrm{MHz}, \mathrm{CDCl}_{3}\right)$








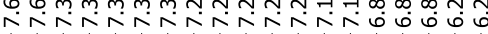

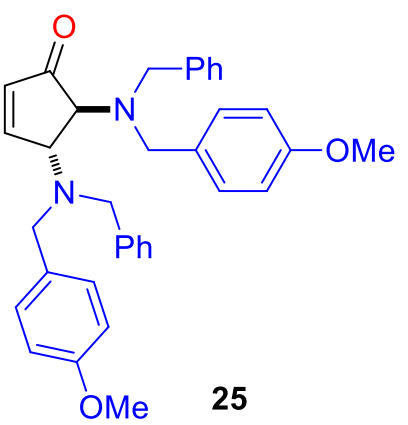

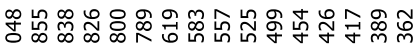

${ }^{1} \mathrm{H}$ NMR $\left(500 \mathrm{MHz}, \mathrm{CDCl}_{3}\right)$
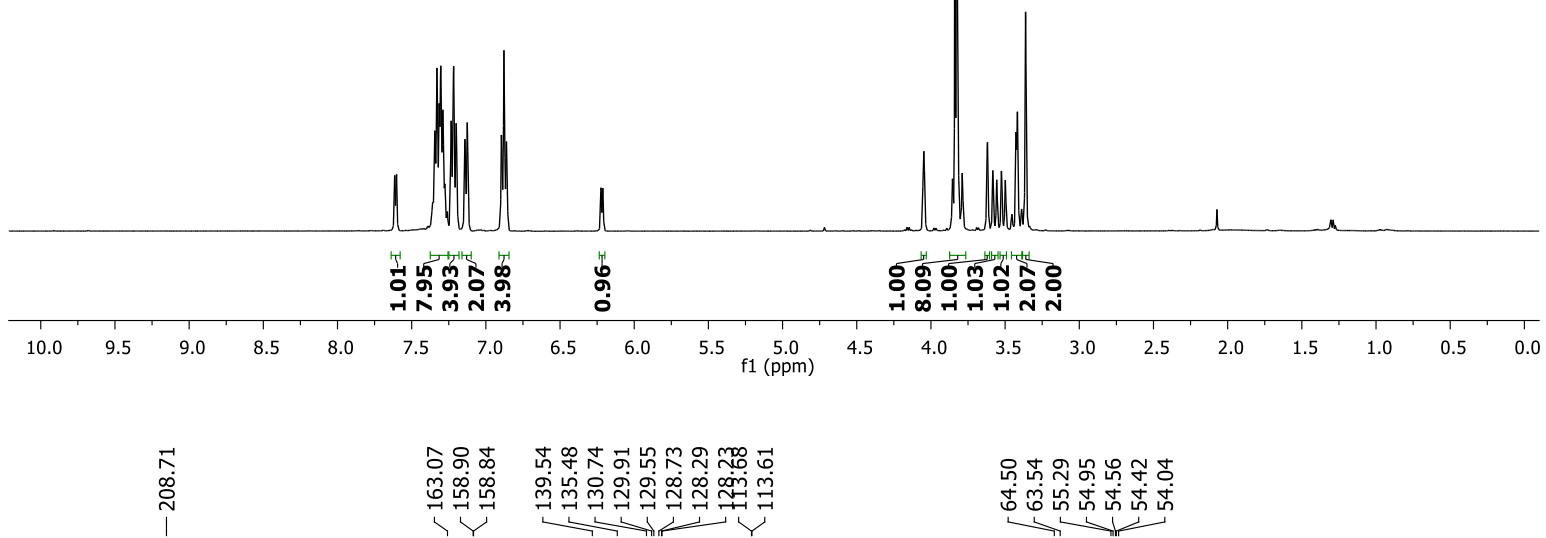

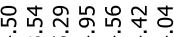

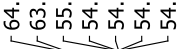

${ }^{13} \mathrm{C}\left\{{ }^{1} \mathrm{H}\right\}$ NMR $\left(125 \mathrm{MHz}, \mathrm{CDCl}_{3}\right)$






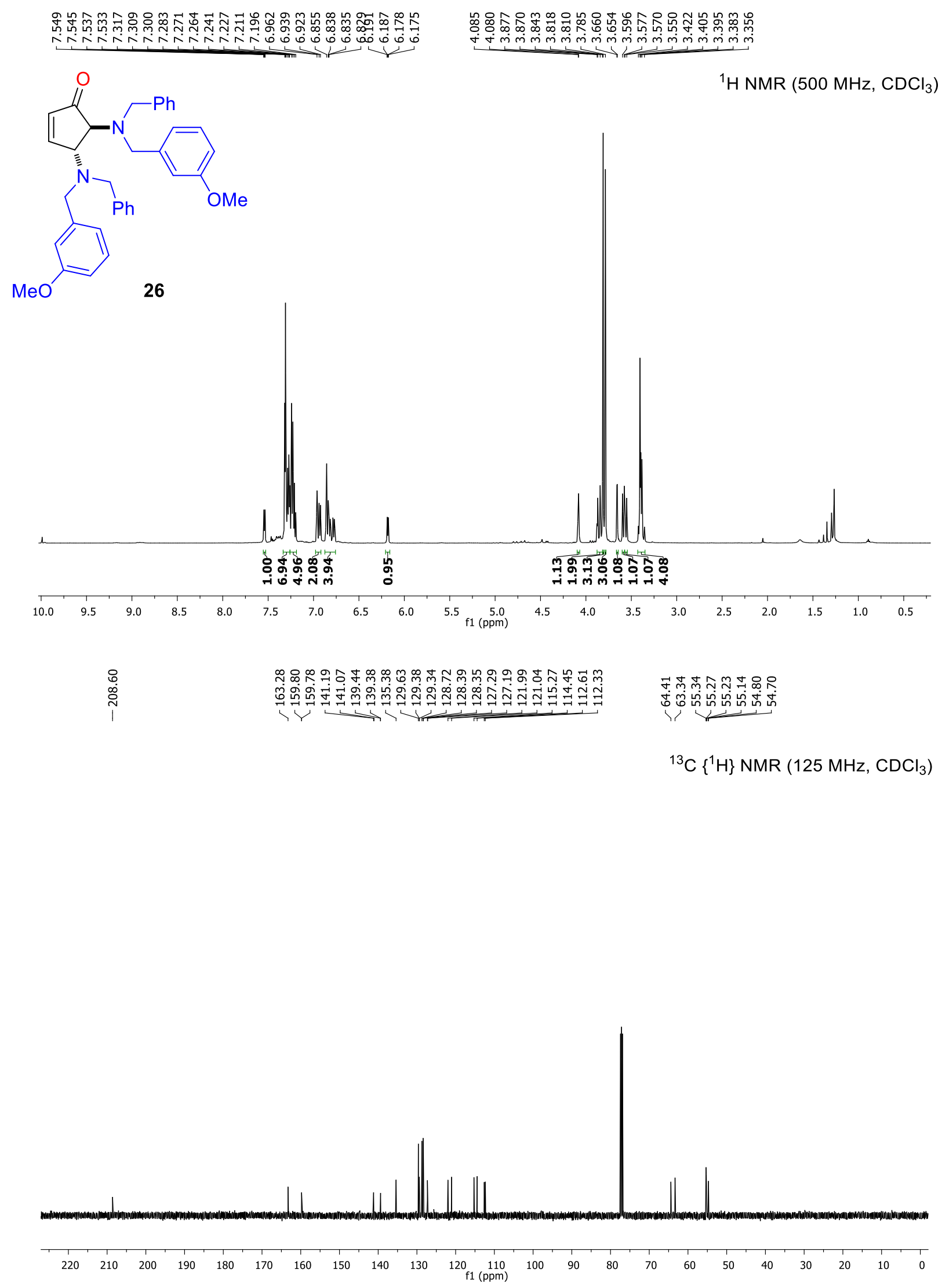


<smiles>O=C1C=C[C@@H](N(Cc2ccccc2)Cc2ccco2)[C@H]1N(Cc1ccccc1)Cc1ccco1</smiles>
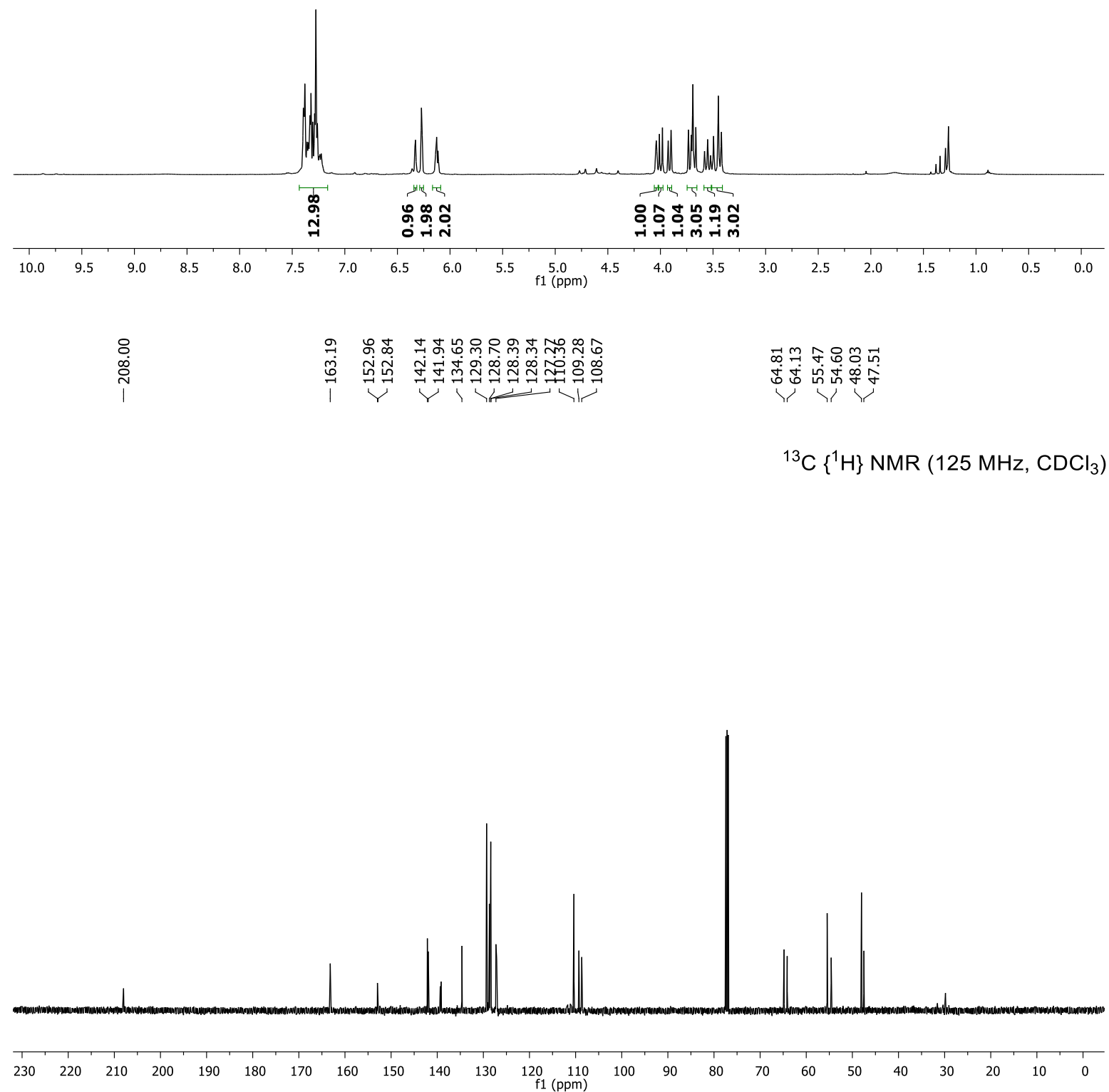
<smiles>CCC(C)CN(Cc1ccccc1)C1C(=O)C=C[C@@H]1CC</smiles>

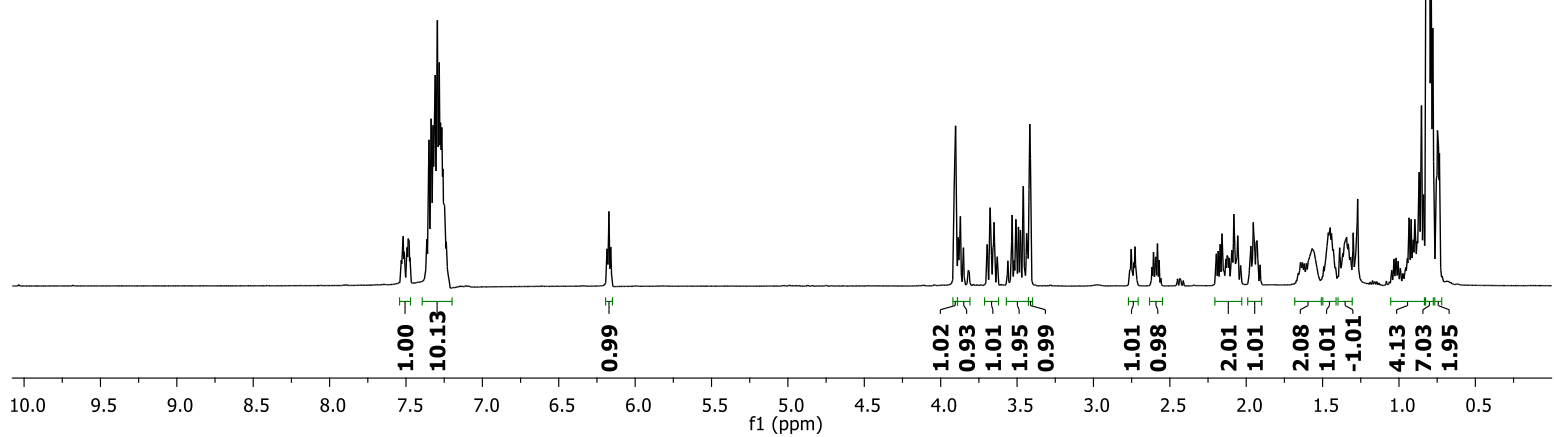



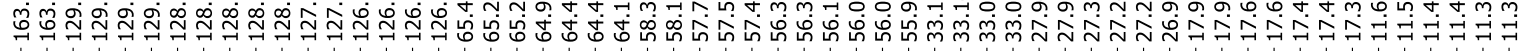

${ }^{13} \mathrm{C}\left\{{ }^{1} \mathrm{H}\right\}$ NMR $\left(125 \mathrm{MHz}, \mathrm{CDCl}_{3}\right)$

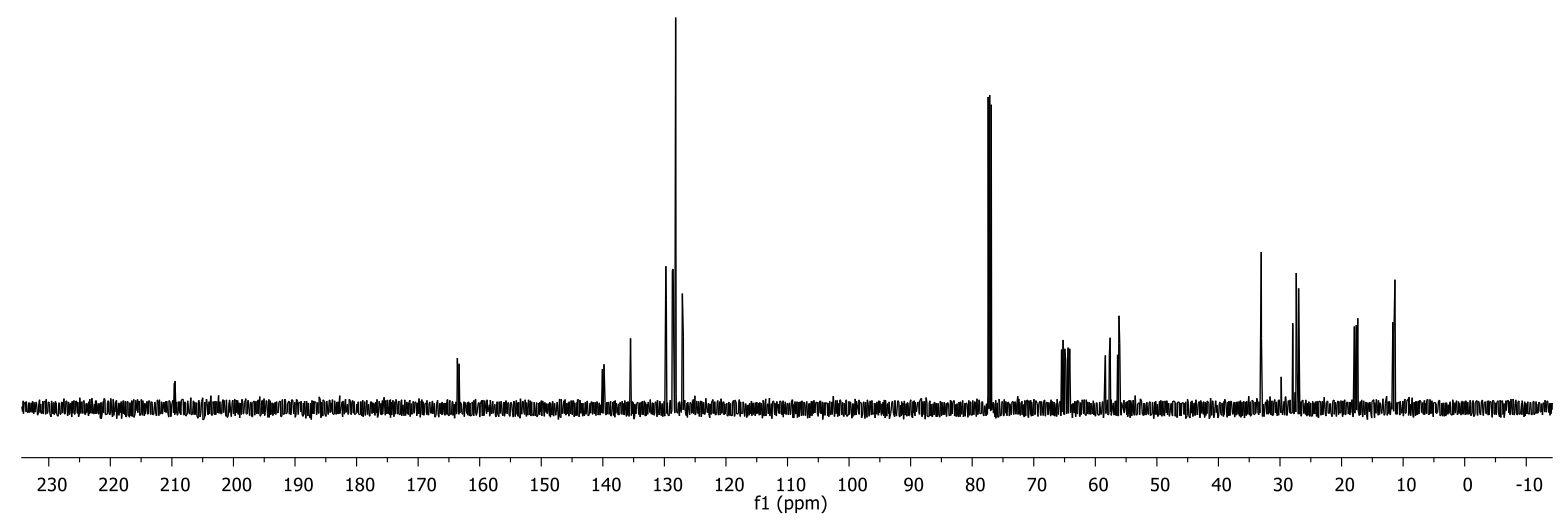




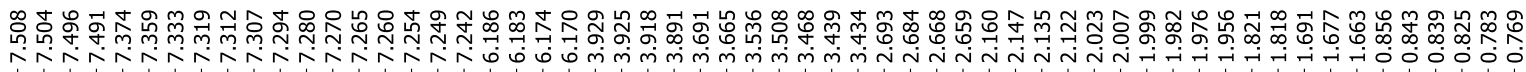<smiles>CC(C)CN(Cc1ccccc1)C1CC(=O)C=C1C(C)C</smiles>

${ }^{1} \mathrm{H}$ NMR $\left(500 \mathrm{MHz}, \mathrm{CDCl}_{3}\right)$
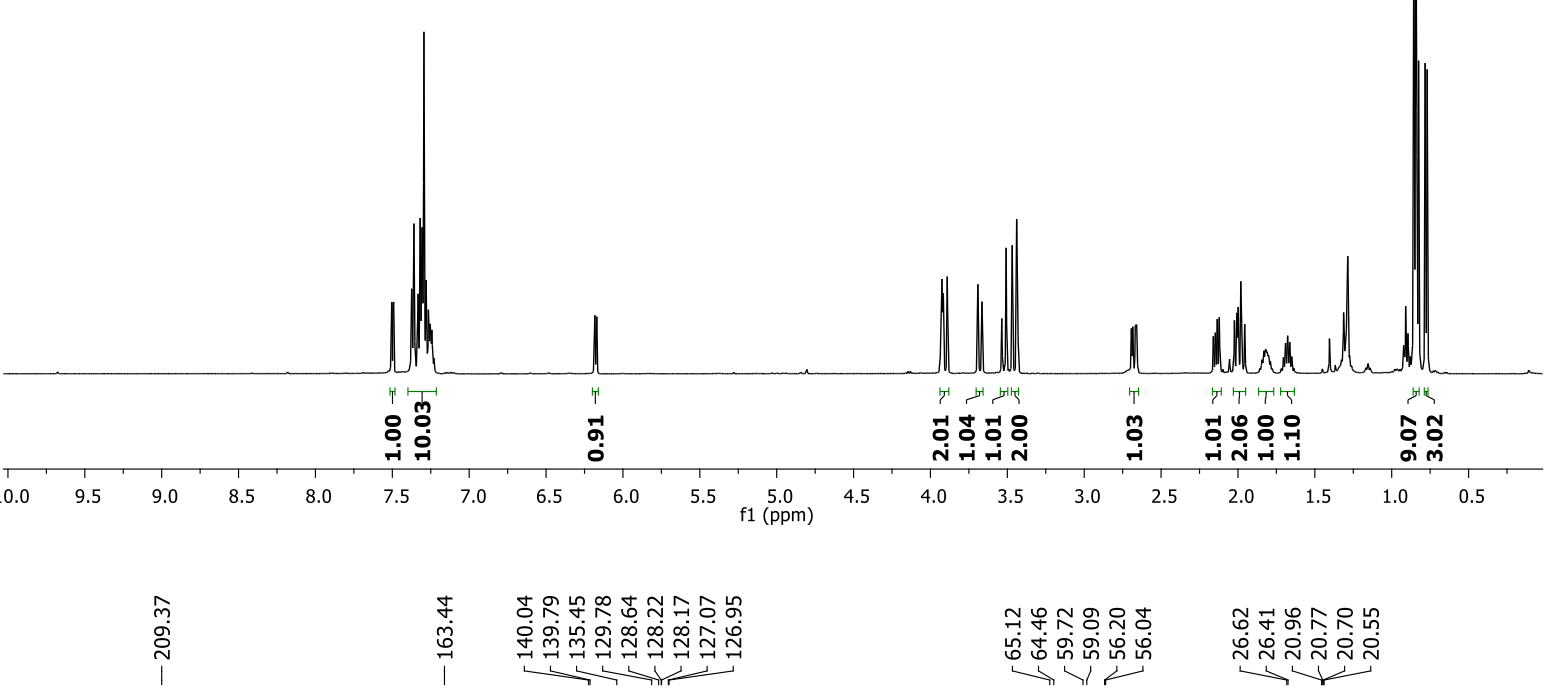

${ }^{13} \mathrm{C}\left\{{ }^{1} \mathrm{H}\right\} \mathrm{NMR}\left(125 \mathrm{MHz}, \mathrm{CDCl}_{3}\right)$








<smiles>CCCCN(Cc1ccc(C)cc1)C1C(=O)C=C[C@@H]1N(CCCC)Cc1ccc(C)cc1</smiles>

${ }^{1} \mathrm{H}$ NMR $\left(500 \mathrm{MHz}, \mathrm{CDCl}_{3}\right)$

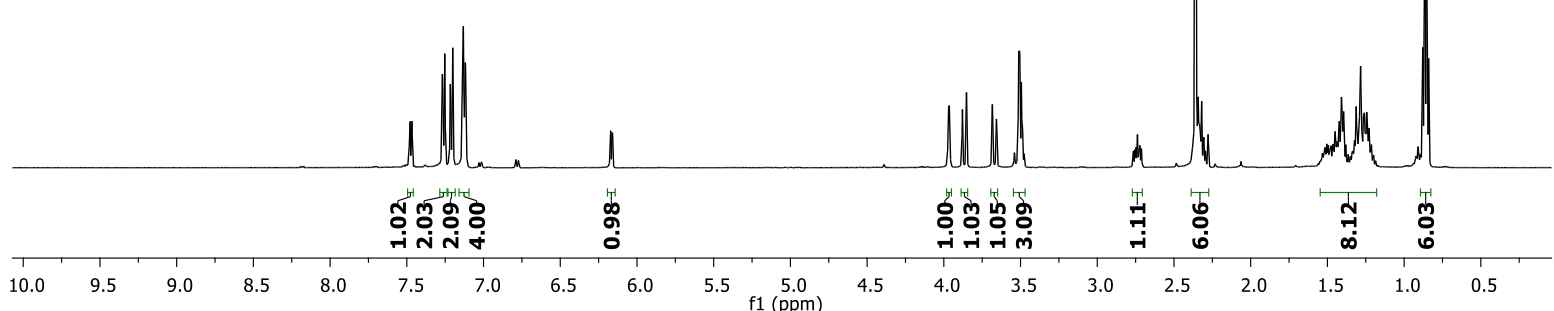



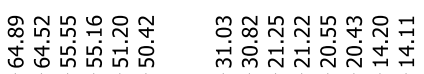

${ }^{13} \mathrm{C}\left\{{ }^{1} \mathrm{H}\right\}$ NMR $\left(125 \mathrm{MHz}, \mathrm{CDCl}_{3}\right)$






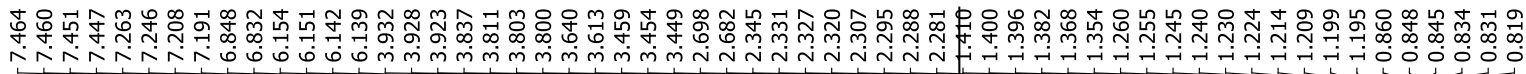<smiles>CCCCN(Cc1ccc(OC)cc1)[C@@H]1C(=O)C=C[C@H]1N(CCCC)Cc1ccc(OC)cc1</smiles>

${ }^{1} \mathrm{H}$ NMR $\left(500 \mathrm{MHz}, \mathrm{CDCl}_{3}\right)$
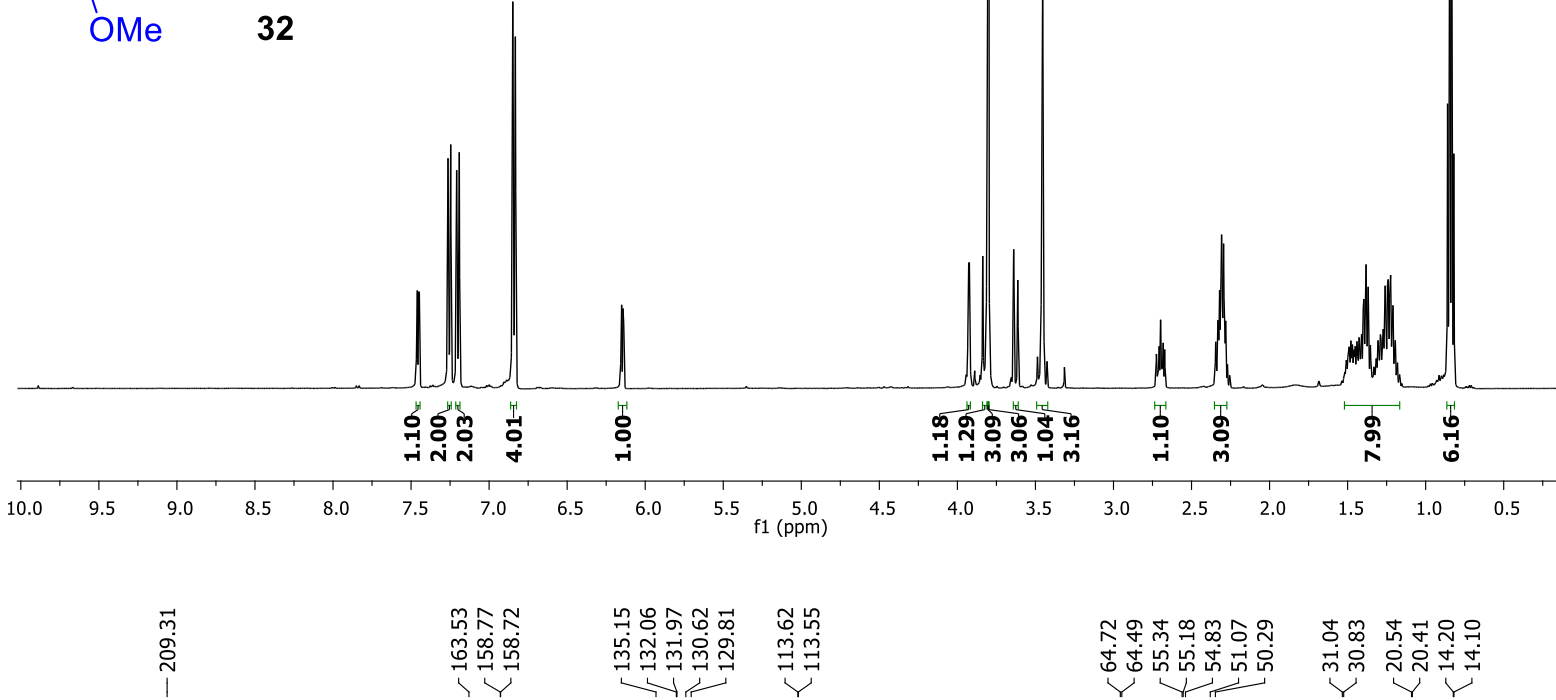

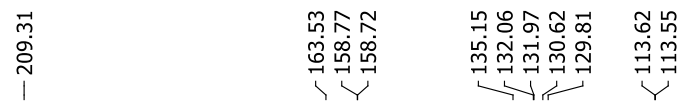

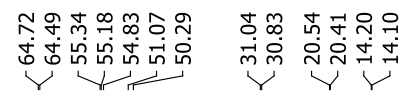

${ }^{13} \mathrm{C}\left\{{ }^{1} \mathrm{H}\right\}$ NMR $\left(125 \mathrm{MHz}, \mathrm{CDCl}_{3}\right)$






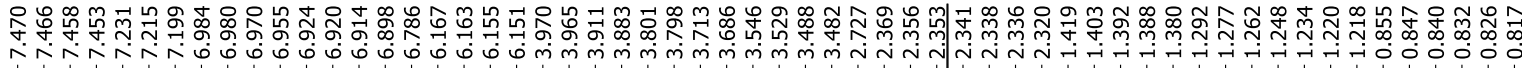<smiles>CCCCN(Cc1cccc(OC)c1)C1C(=O)C=C[C@@H]1N(CCCC)Cc1cccc(OC)c1</smiles>

${ }^{1} \mathrm{H} \mathrm{NMR}\left(500 \mathrm{MHz}, \mathrm{CDCl}_{3}\right)$

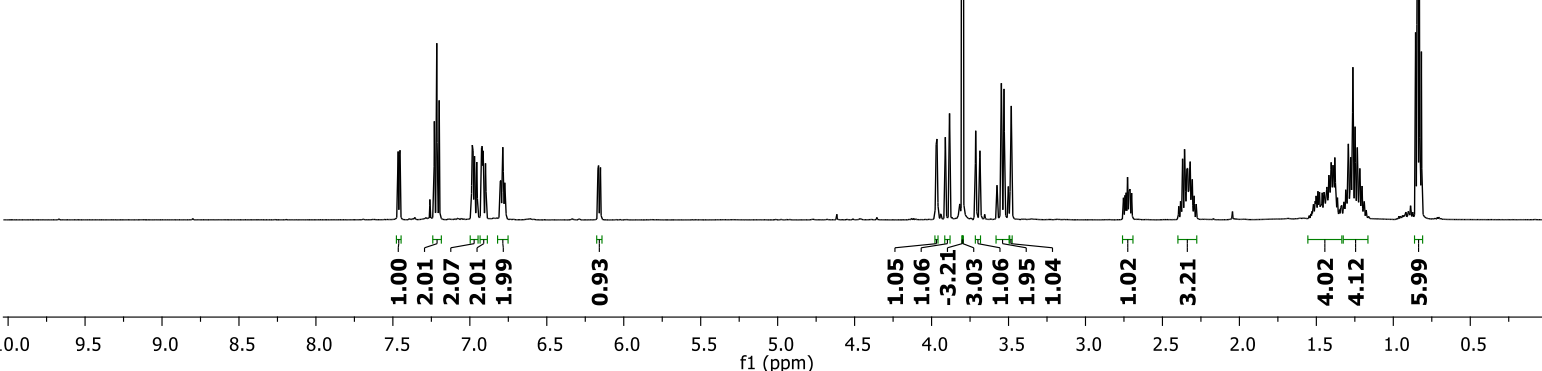

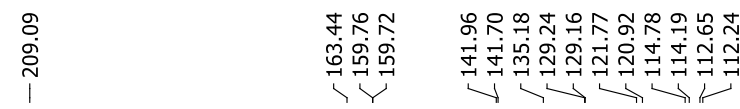

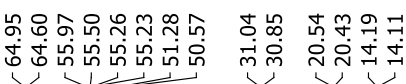

${ }^{13} \mathrm{C}\left\{{ }^{1} \mathrm{H}\right\}$ NMR $\left(125 \mathrm{MHz}, \mathrm{CDCl}_{3}\right)$

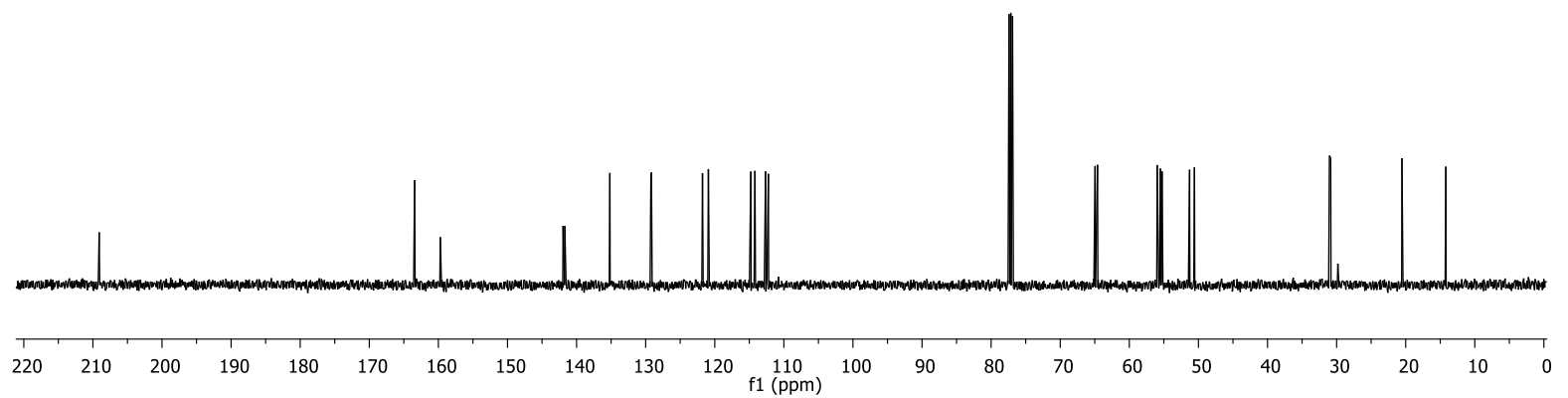



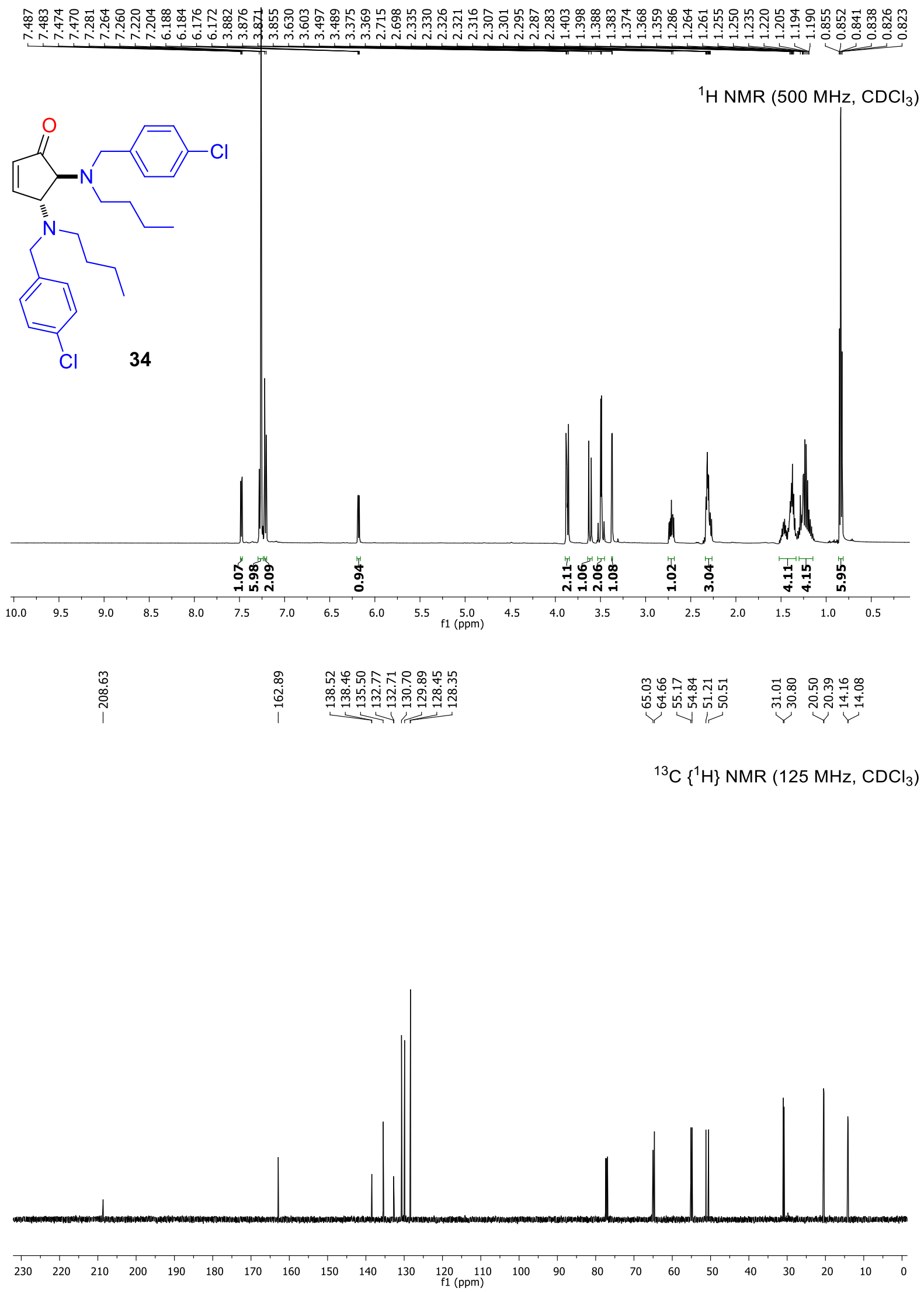


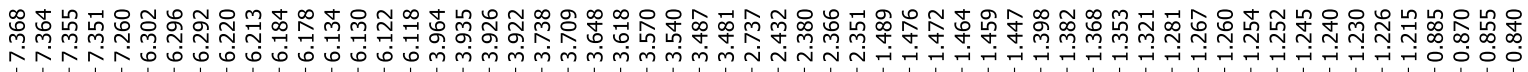<smiles>CCCCN(Cc1ccco1)[C@@H]1C(=O)C=C[C@H]1N(CCCC)Cc1ccco1</smiles>

${ }^{1} \mathrm{H}$ NMR $\left(500 \mathrm{MHz}, \mathrm{CDCl}_{3}\right)$

<smiles>CCCCN(Cc1cccs1)[C@@H]1C=CC(=O)[C@H]1Cc1cccs1</smiles>

${ }^{1} \mathrm{H}$ NMR $\left(500 \mathrm{MHz}, \mathrm{CDCl}_{3}\right)$
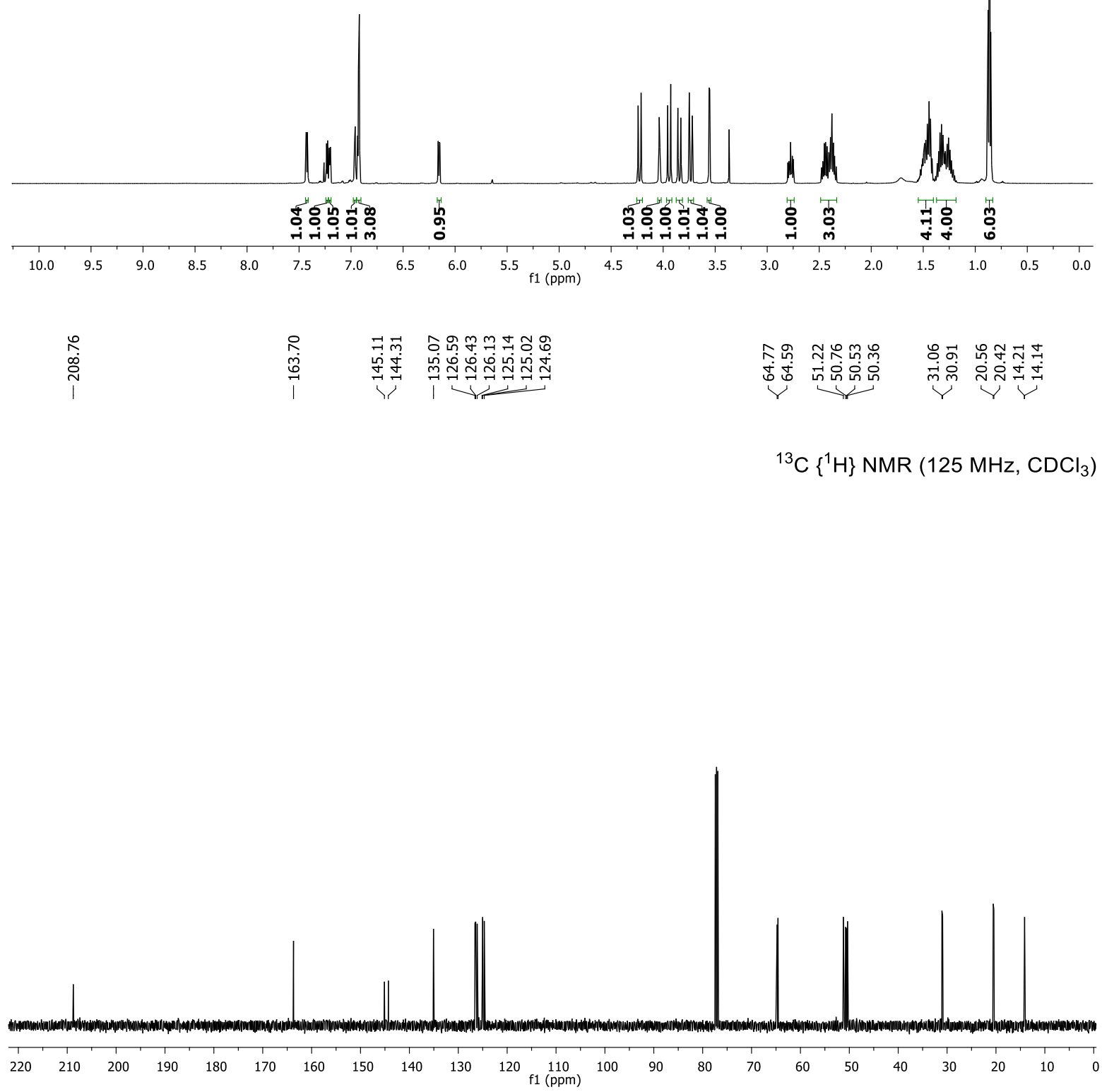


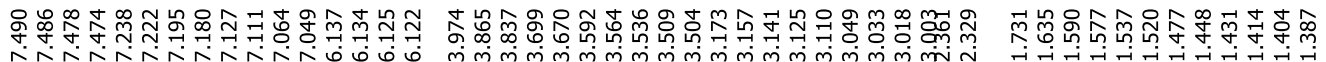

${ }^{1} \mathrm{H} \mathrm{NMR}\left(500 \mathrm{MHz}, \mathrm{CDCl}_{3}\right)$<smiles>Cc1ccc(CN(C2CCCC2)C2C(=O)C=C[C@@H]2N(Cc2ccc(C)cc2)C2CCCC2)cc1</smiles>



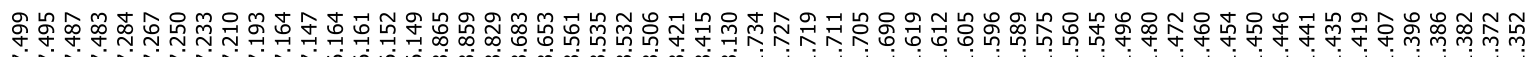

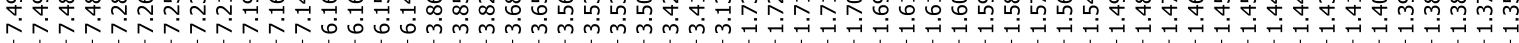<smiles>O=C1C=C[C@@H](N(Cc2ccc(Cl)cc2)C2CCCC2)C1N(Cc1ccc(Cl)cc1)C1CCCC1</smiles>

${ }^{1} \mathrm{H}$ NMR $\left(500 \mathrm{MHz}, \mathrm{CDCl}_{3}\right)$

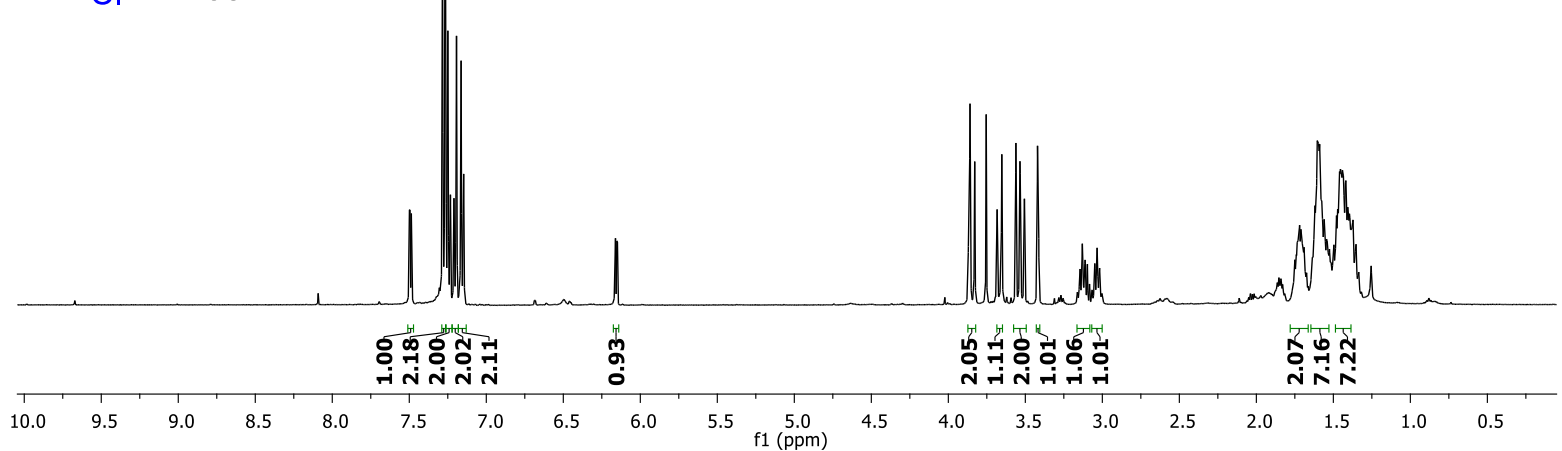

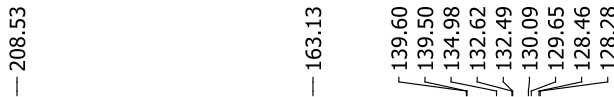

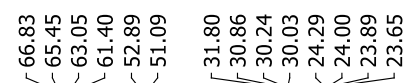

mm m N N N

${ }^{13} \mathrm{C}\left\{{ }^{1} \mathrm{H}\right\}$ NMR $\left(125 \mathrm{MHz}, \mathrm{CDCl}_{3}\right)$

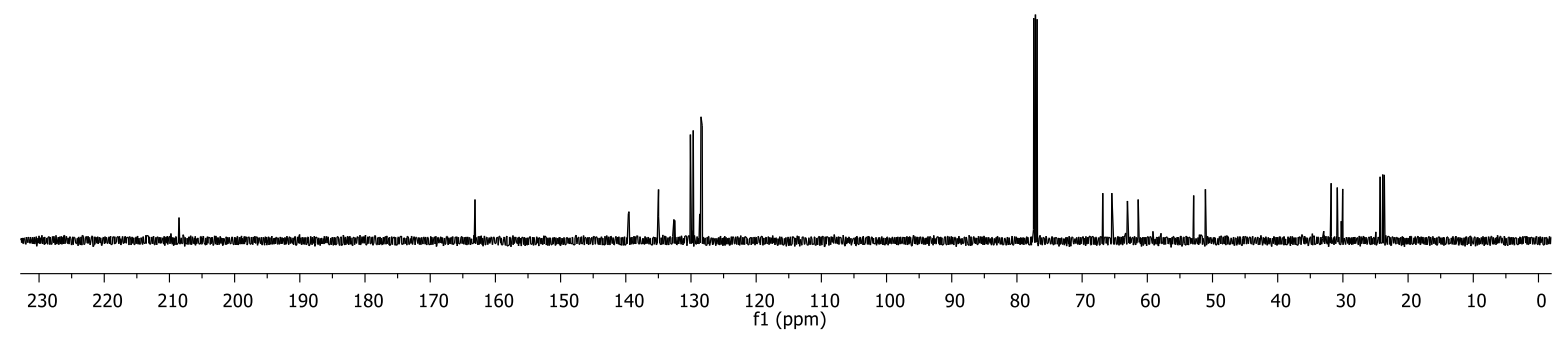




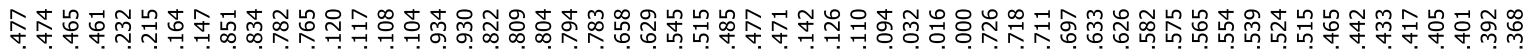

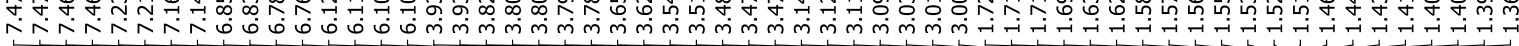<smiles>COc1ccc(CN(C2CCCC2)[C@@H]2C(=O)C=C[C@H]2N(Cc2ccc(OC)cc2)C2CCCC2)cc1</smiles>

${ }^{1} \mathrm{H}$ NMR $\left(500 \mathrm{MHz}, \mathrm{CDCl}_{3}\right)$



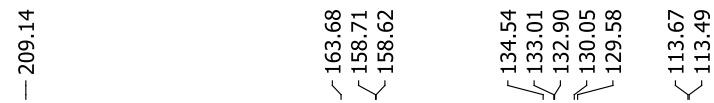

ร 궁ำ



${ }^{13} \mathrm{C}\left\{{ }^{1} \mathrm{H}\right\}$ NMR $\left(125 \mathrm{MHz}, \mathrm{CDCl}_{3}\right)$

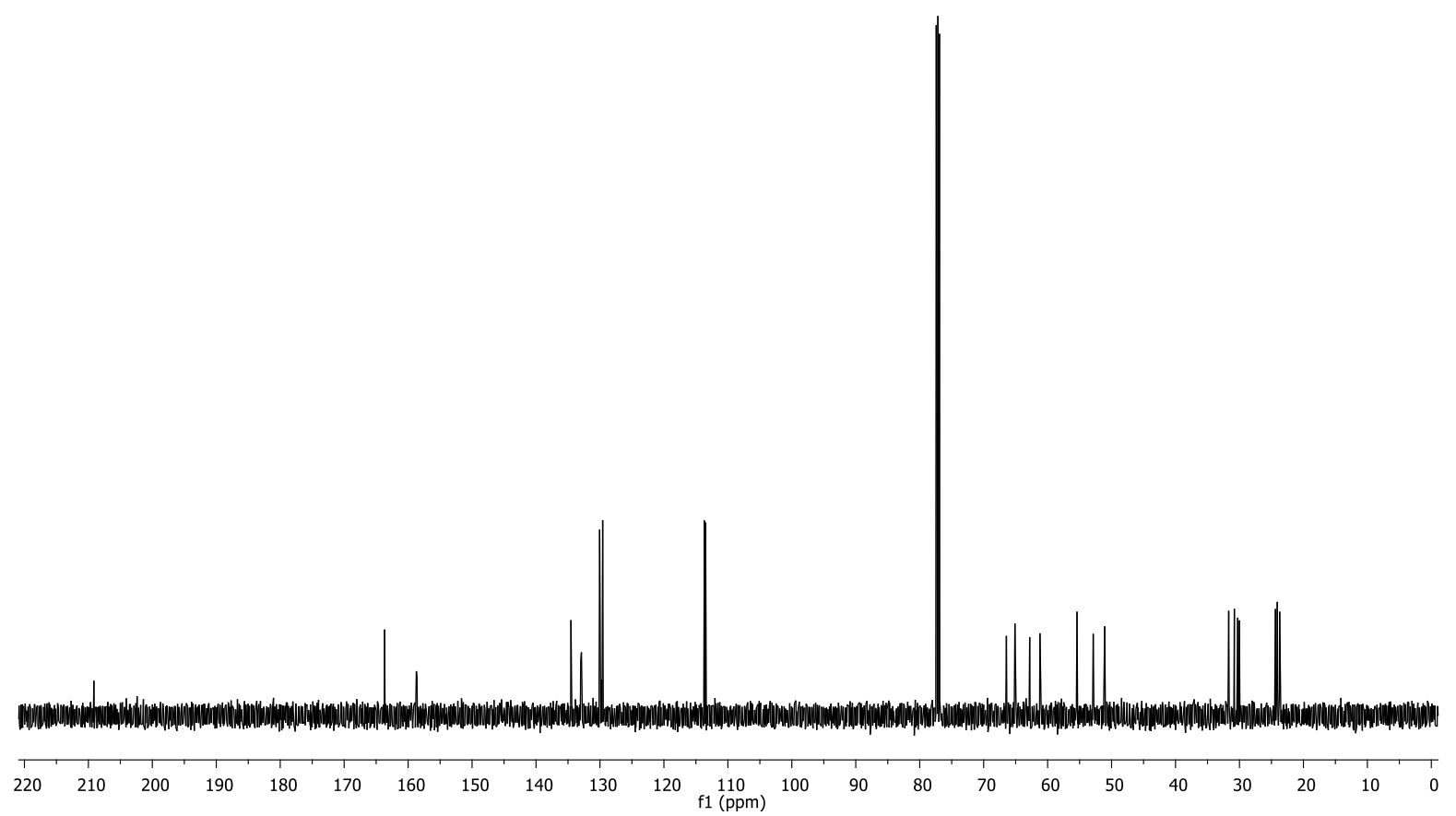



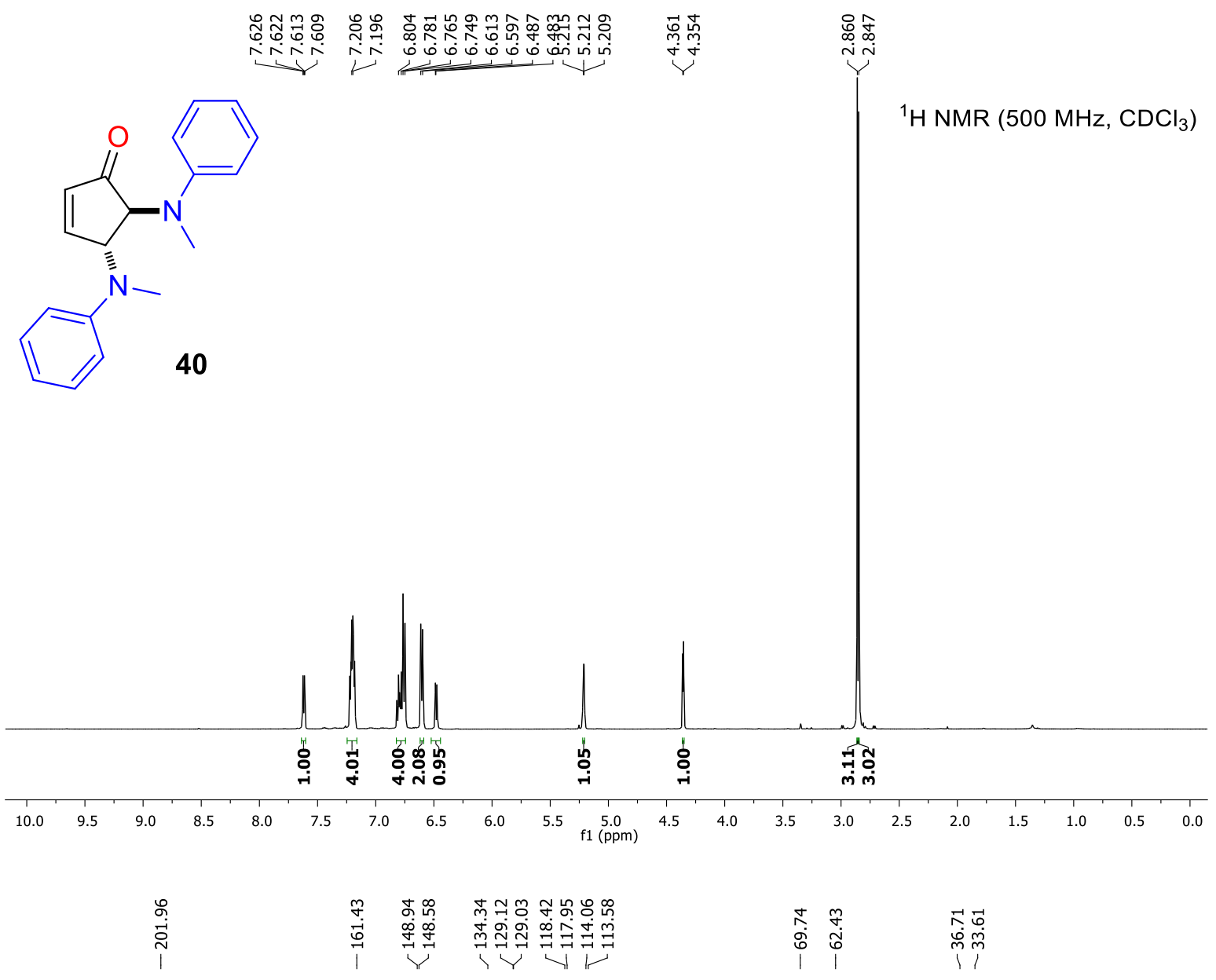

${ }^{13} \mathrm{C}\left\{{ }^{1} \mathrm{H}\right\}$ NMR $\left(125 \mathrm{MHz}, \mathrm{CDCl}_{3}\right)$

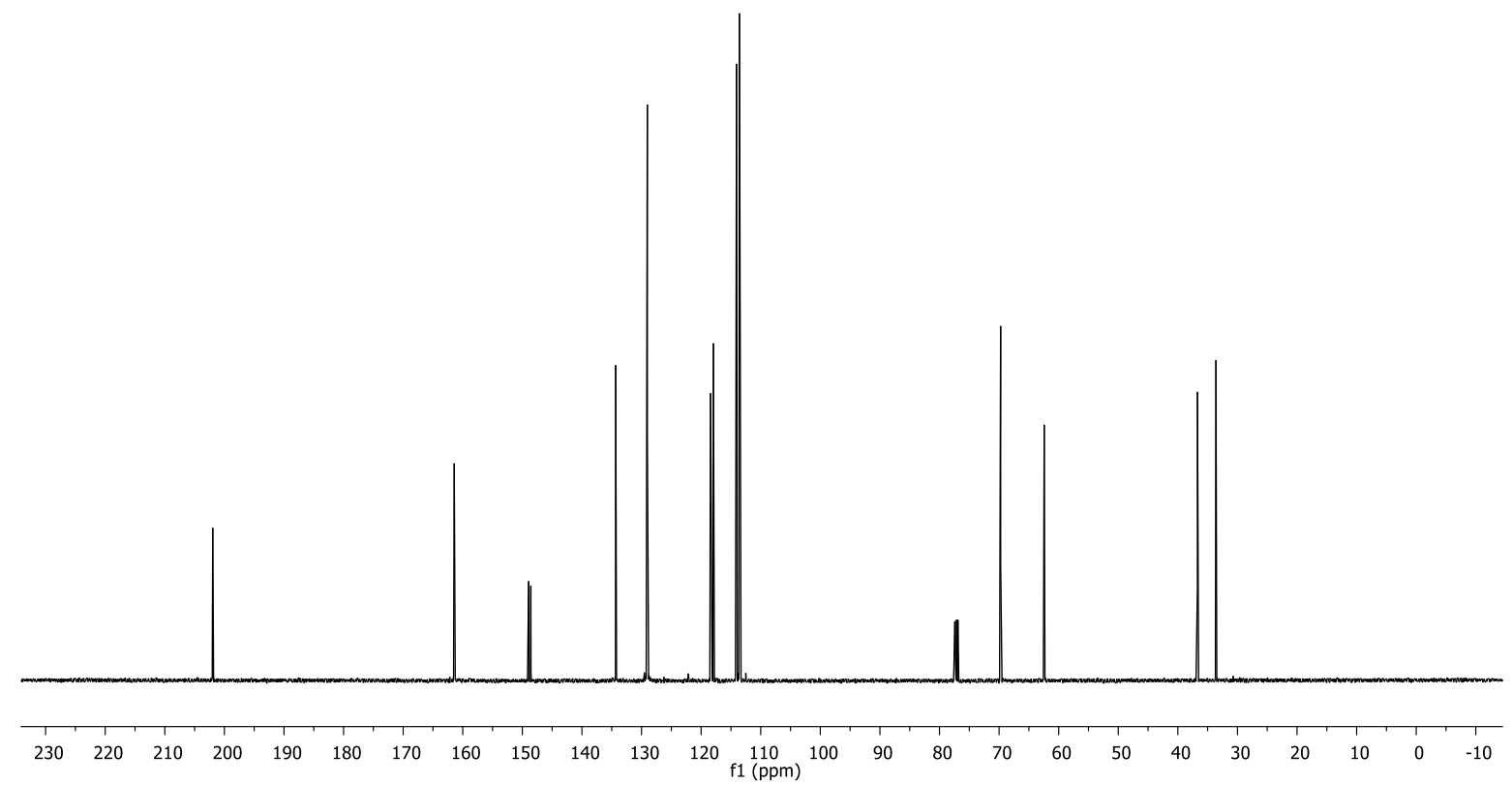


Copies of ${ }^{1} \mathrm{H}$ and ${ }^{13} \mathrm{C}\{\mathrm{H}\}$ NMR spectra of trans-DACPs from substituted anilines
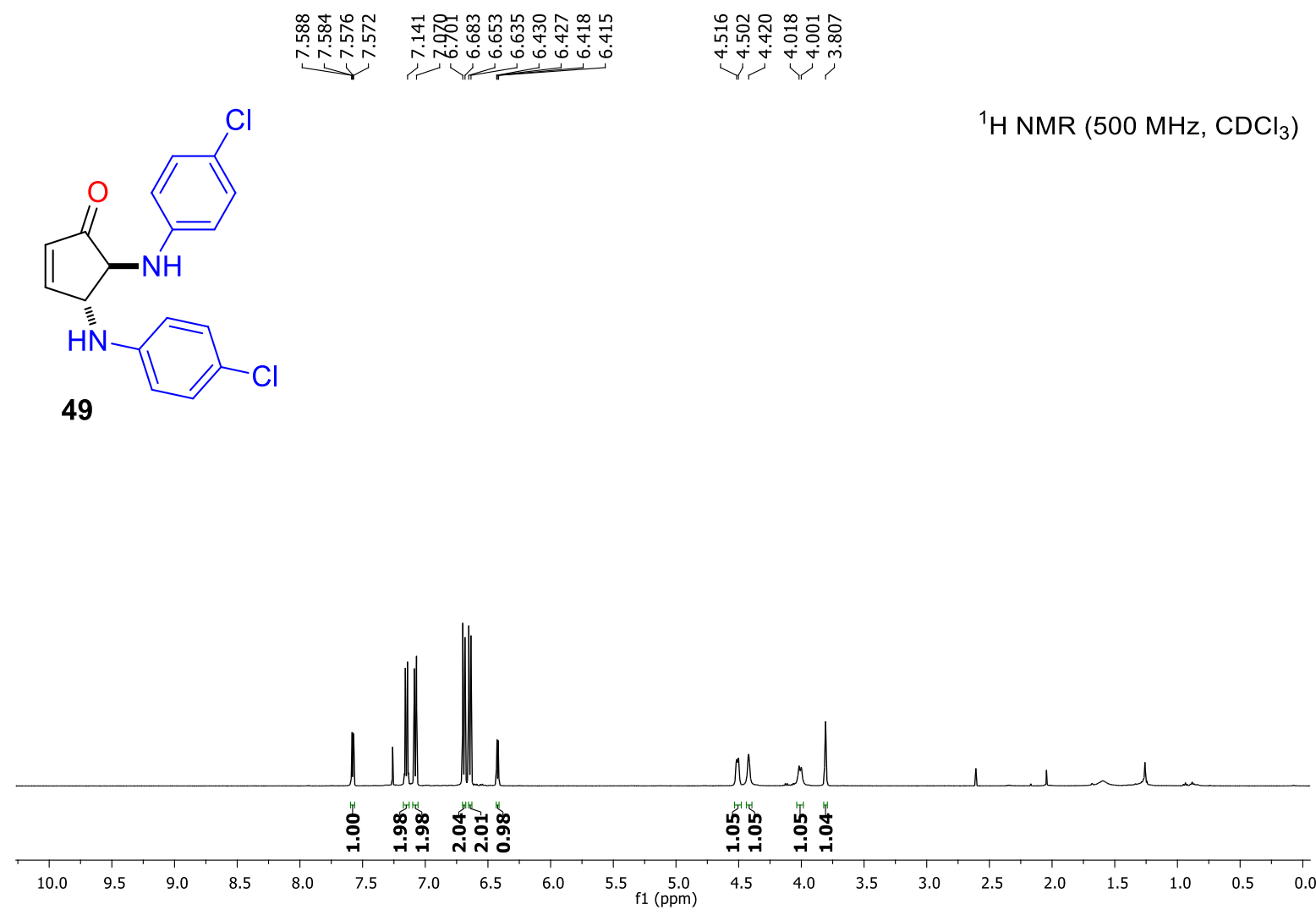



${ }^{13} \mathrm{C}\left\{{ }^{1} \mathrm{H}\right\} \operatorname{NMR}\left(125 \mathrm{MHz}, \mathrm{CDCl}_{3}\right)$

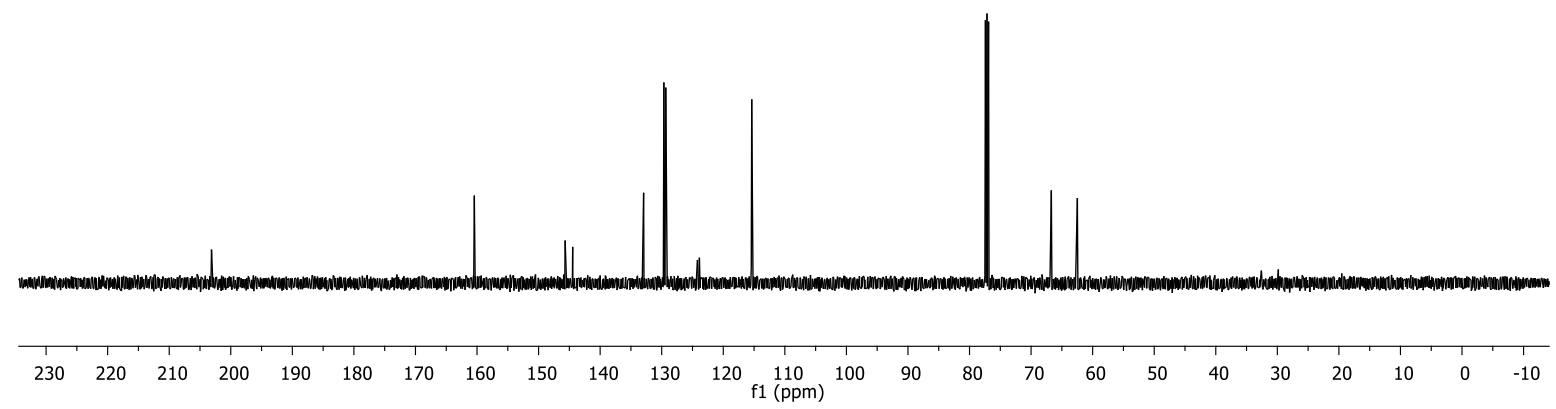


<smiles>O=C1C=C[C@@H](Nc2ccccc2Cl)[C@@H]1Nc1ccccc1Cl</smiles>





${ }^{13} \mathrm{C}\left\{{ }^{1} \mathrm{H}\right\}$ NMR $\left(125 \mathrm{MHz}, \mathrm{CDCl}_{3}\right)$










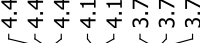

${ }^{1} \mathrm{H}$ NMR $\left(500 \mathrm{MHz}, \mathrm{CDCl}_{3}\right)$<smiles>O=C1C=C[C@@H](Nc2ccc(Br)cc2)[C@@H]1Nc1ccc(Br)cc1</smiles>

52

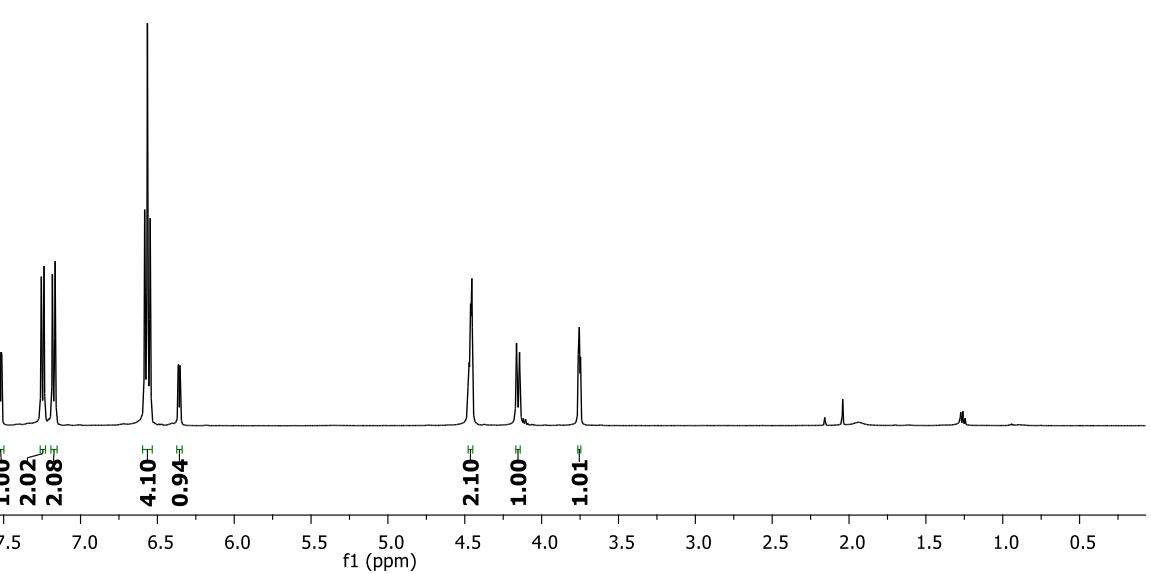

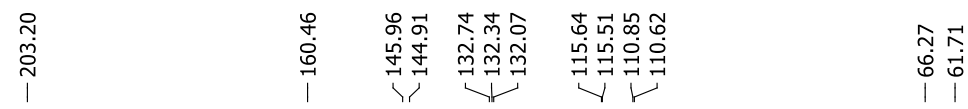

${ }^{13} \mathrm{C}\left\{{ }^{1} \mathrm{H}\right\}$ NMR $\left(125 \mathrm{MHz}, \mathrm{CDCl}_{3}\right)$








53

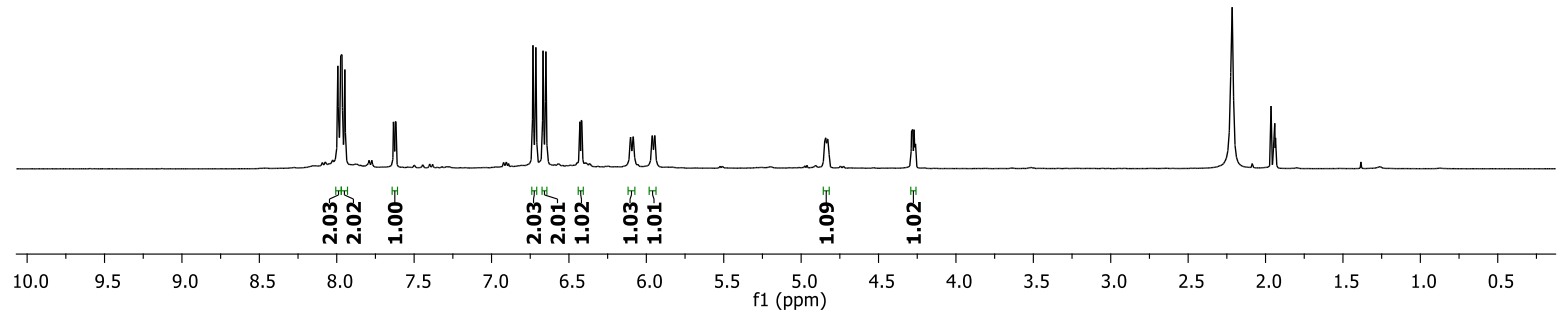

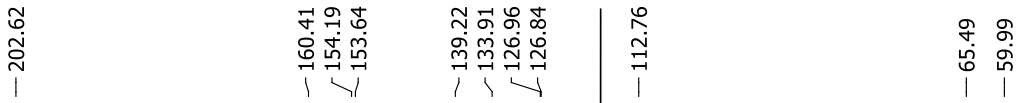

${ }^{13} \mathrm{C}\left\{{ }^{1} \mathrm{H}\right\}$ NMR $\left(125 \mathrm{MHz}, \mathrm{CD}_{3} \mathrm{CN}\right)$

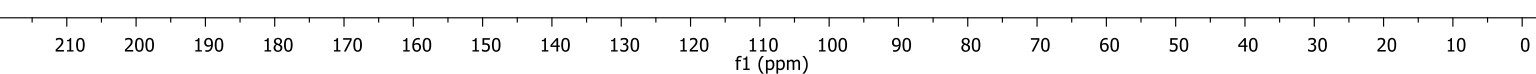



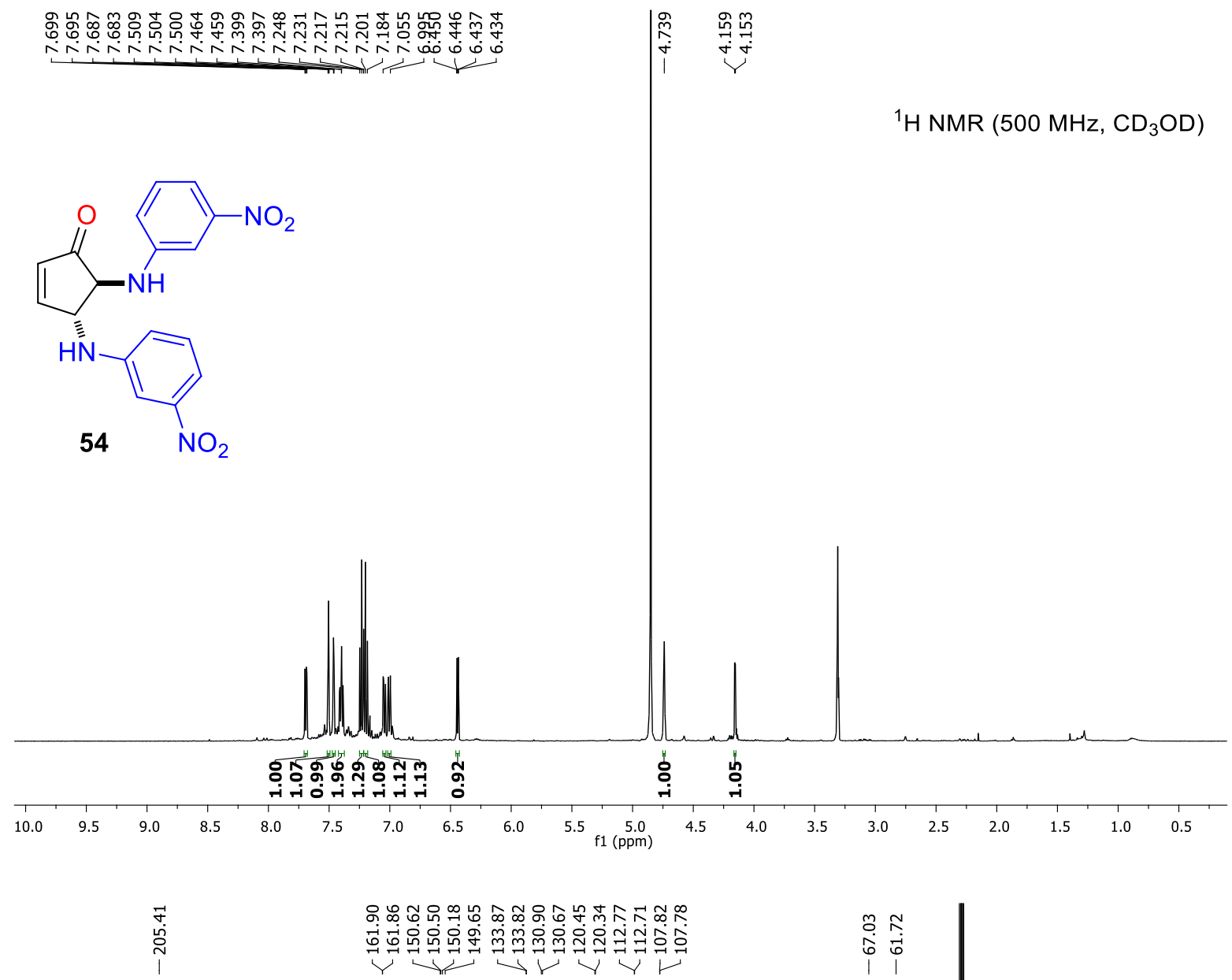

${ }^{13} \mathrm{C}\left\{{ }^{1} \mathrm{H}\right\}$ NMR $\left(125 \mathrm{MHz}, \mathrm{CD}_{3} \mathrm{OD}\right)$

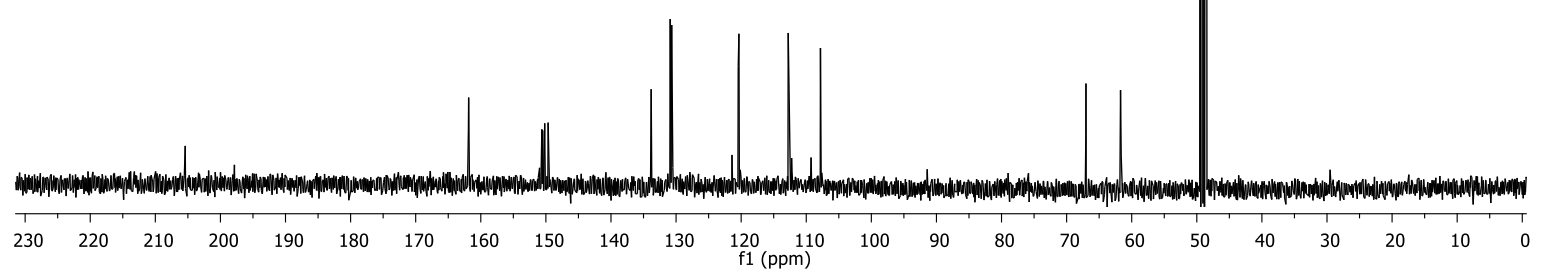






Li்<smiles>CC(=O)c1ccc(NC2CC=CC2=O)cc1</smiles><smiles>CNc1ccc(C(C)=O)cc1</smiles>

55



${ }^{1} \mathrm{H}$ NMR $\left(500 \mathrm{MHz}, \mathrm{CDCl}_{3}\right)$

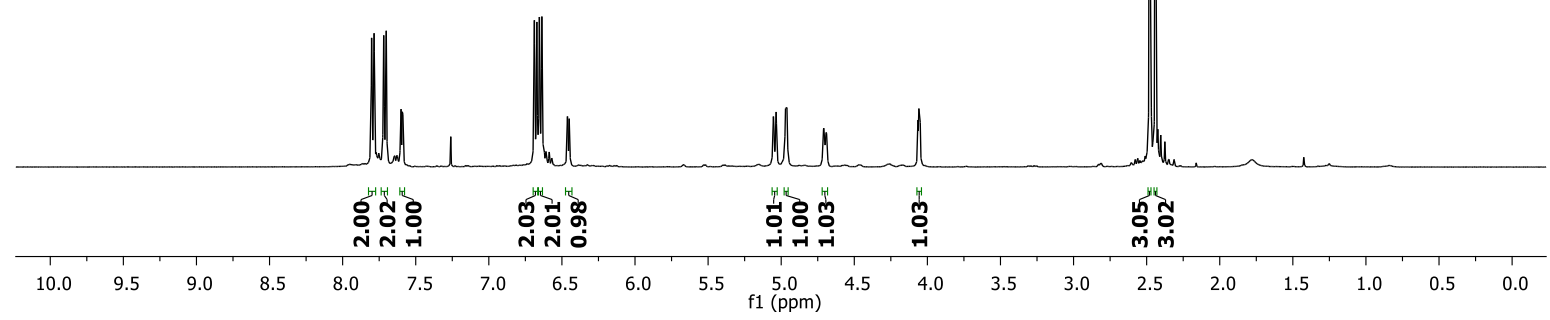

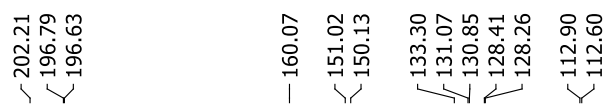

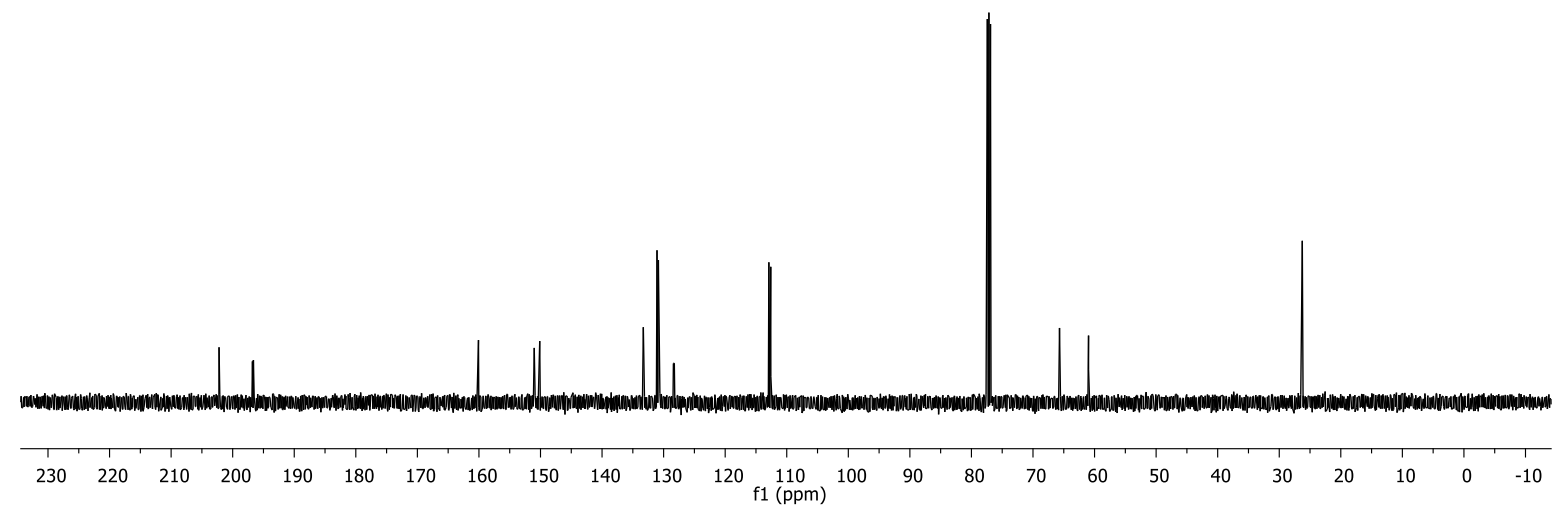


${ }^{1} \mathrm{H}$ NMR $\left(500 \mathrm{MHz}, \mathrm{CDCl}_{3}\right)$<smiles>CCOC(=O)c1ccc(N[C@@H]2C(=O)C=C[C@H]2Nc2ccc(C(=O)OCC)cc2)cc1</smiles>
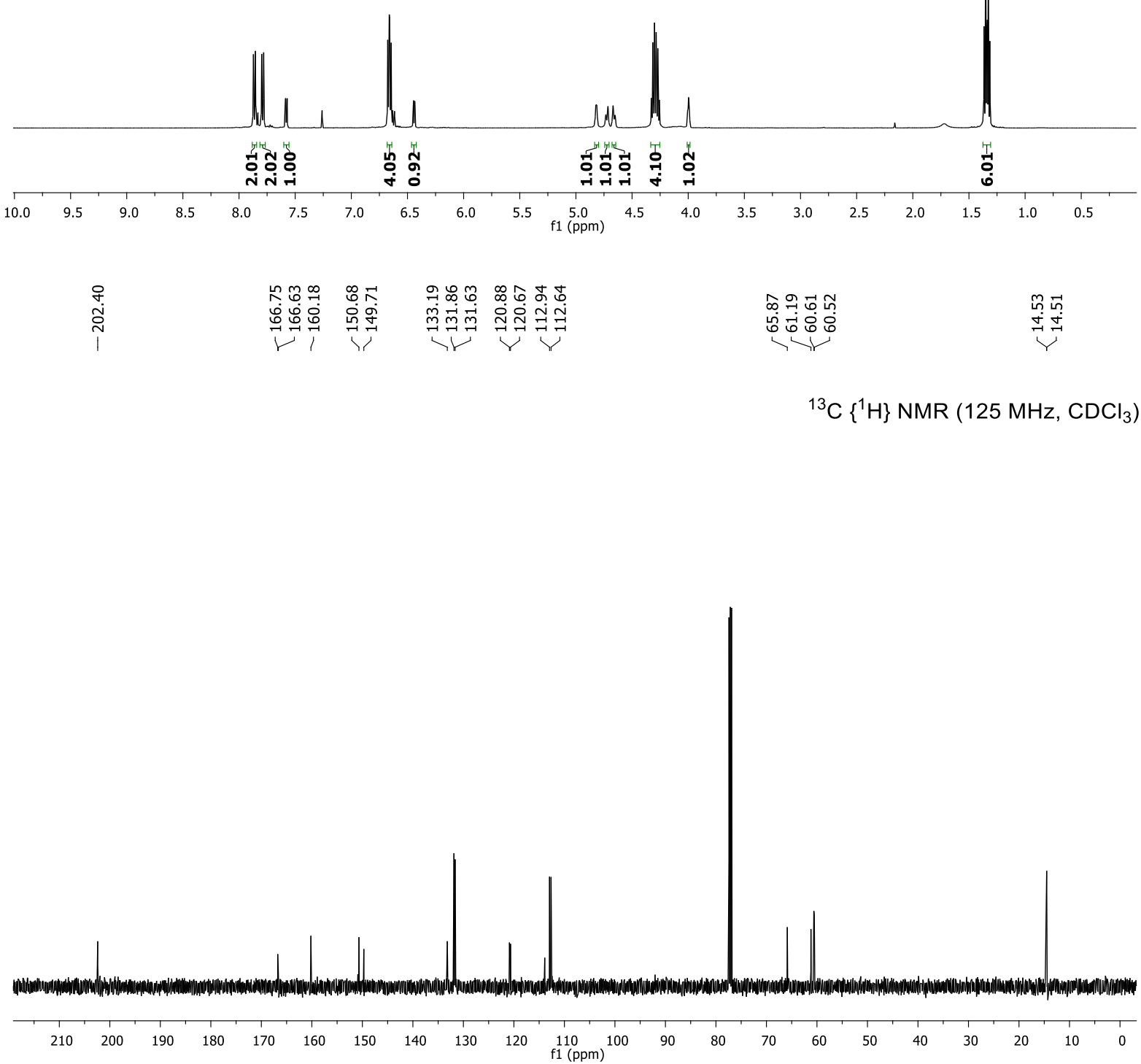
${ }^{1} \mathrm{H}$ NMR $\left(500 \mathrm{MHz}\right.$, DMSO-d $\left.{ }_{6}\right)$<smiles>O=C(O)c1ccccc1NC1C(=O)C=C[C@@H]1Nc1ccccc1C(=O)O</smiles>

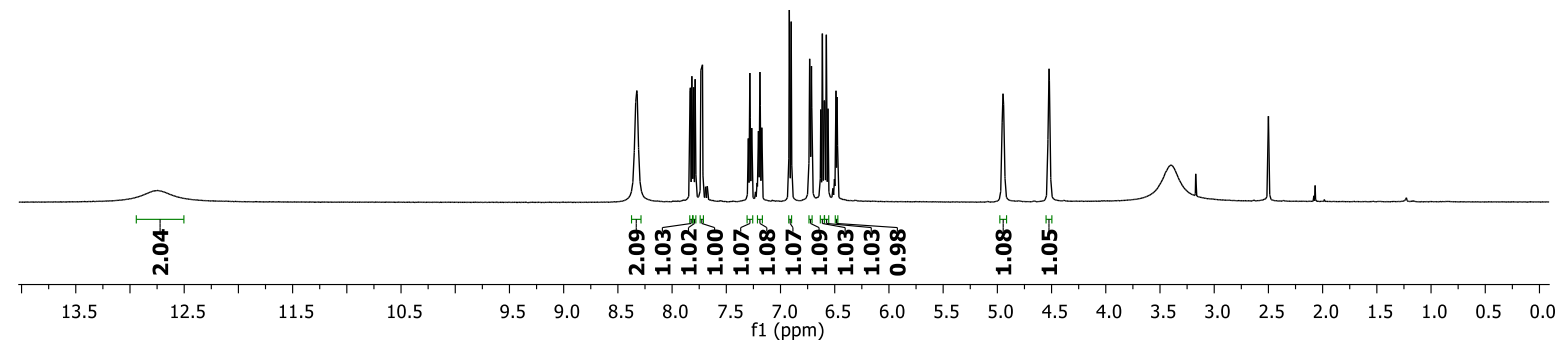

${ }^{13} \mathrm{C}\left\{{ }^{1} \mathrm{H}\right\}$ NMR (125 MHz, DMSO- $\left.d_{6}\right)$






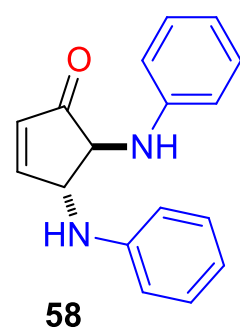

${ }^{1} \mathrm{H}$ NMR $\left(500 \mathrm{MHz}, \mathrm{CDCl}_{3}\right)$

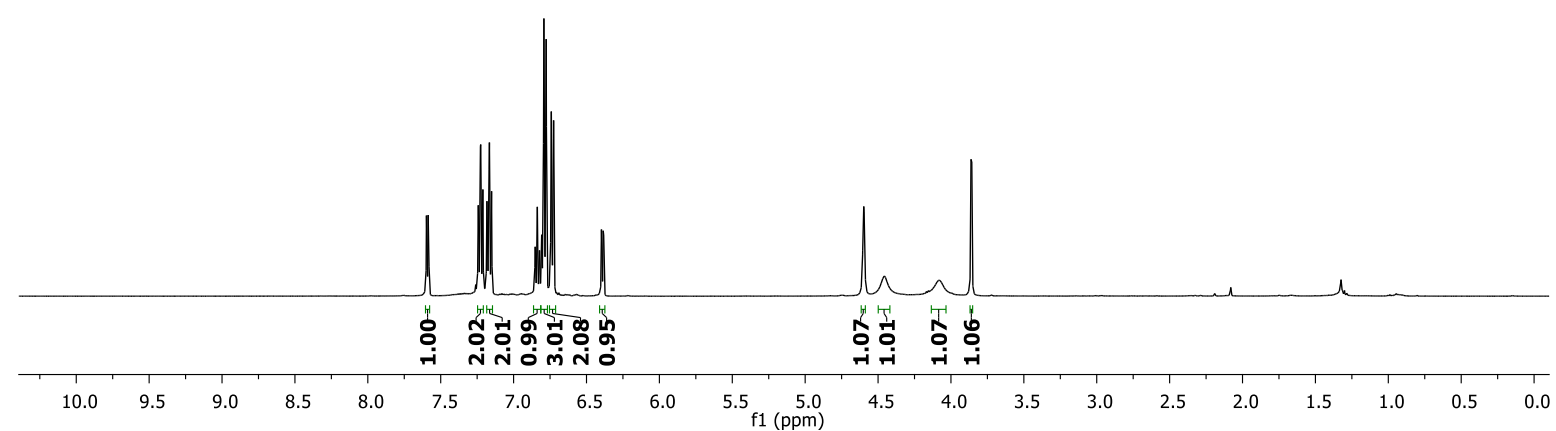



${ }^{13} \mathrm{C}\left\{{ }^{1} \mathrm{H}\right\}$ NMR $\left(125 \mathrm{MHz}, \mathrm{CDCl}_{3}\right)$

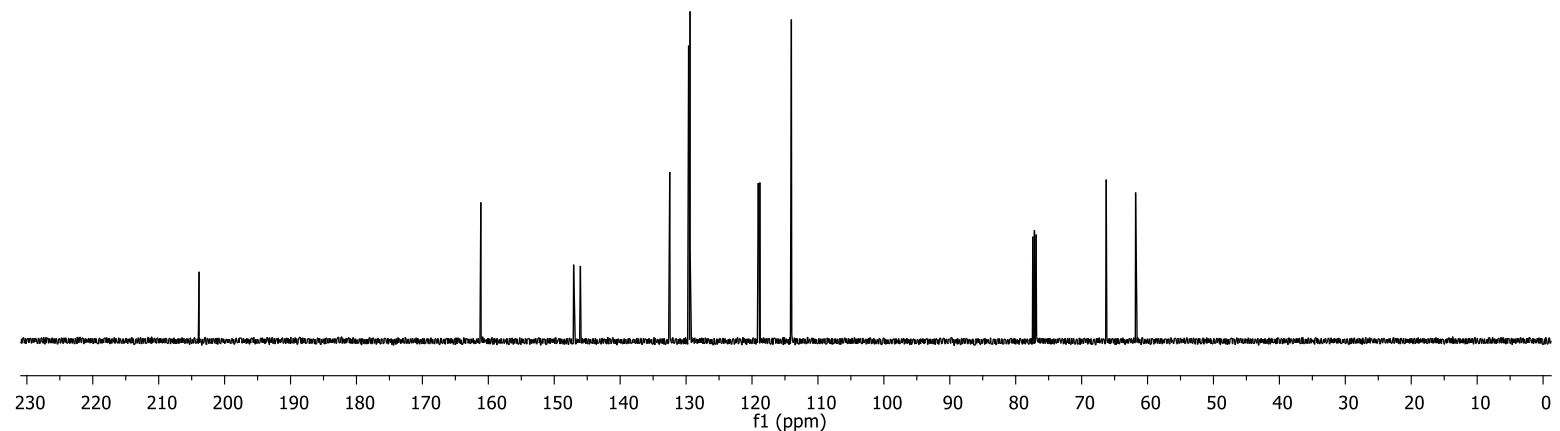


${ }^{1} \mathrm{H}$ NMR $\left(500 \mathrm{MHz}, \mathrm{CDCl}_{3}\right)$
